\title{
Background assessment for the TREX dark matter experiment
}

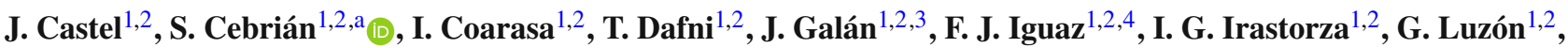 \\ H. Mirallas ${ }^{1,2}$, A. Ortiz de Solórzano ${ }^{1,2}$, E. Ruiz-Chóliz ${ }^{1,2}$ \\ ${ }^{1}$ Laboratorio de Física Nuclear y Astropartículas, Universidad de Zaragoza, Calle Pedro Cerbuna 12, 50009 Zaragoza, Spain \\ ${ }^{2}$ Laboratorio Subterráneo de Canfranc, Paseo de los Ayerbe s/n, 22880 Canfranc Estación, Huesca, Spain \\ ${ }^{3}$ Present Address: Shanghai Laboratory for Particle Physics and Cosmology, INPAC and Department of Physics and Astronomy, Shanghai Jiao \\ Tong University, 200240 Shanghai, China \\ ${ }^{4}$ Present Address: Synchrotron Soleil, BP 48, Saint-Aubin, 91192 Gif-sur-Yvette, France
}

Received: 13 December 2018 / Accepted: 7 September 2019 / Published online: 21 September 2019

(C) The Author(s) 2019

\begin{abstract}
TREX-DM is conceived to look for low-mass Weakly Interacting Massive Particles (WIMPs) using a gas Time Projection Chamber equipped with Micromegas readout planes at the Canfranc Underground Laboratory. The detector can hold in the active volume $\sim 20 l$ of pressurized gas up to $10 \mathrm{bar}$, corresponding to $0.30 \mathrm{~kg}$ of Ar or $0.16 \mathrm{~kg}$ of Ne. The Micromegas are read with a self-triggered acquisition, being thresholds below $0.4 \mathrm{keV}$ (electron equivalent) at reach. A low background level in the lowest energy region is another essential requirement. To assess the expected background, all the relevant sources have been considered, including the measured fluxes of gamma radiation, muons and neutrons at the Canfranc Laboratory, together with the activity of most of the components used in the detector and ancillary systems, obtained in a complete assay program. The background contributions have been simulated by means of a dedicated application based on Geant 4 and a custom-made code for the detector response. The background model developed for the detector presently installed in Canfranc points to levels from 1 to 10 counts $\mathrm{keV}^{-1} \mathrm{~kg}^{-1} \mathrm{day}^{-1}$ in the region of interest, making TREX-DM competitive in the search for low-mass WIMPs. A roadmap to further decrease it down to 0.1 counts $\mathrm{keV}^{-1} \mathrm{~kg}^{-1}$ day $^{-1}$ is underway.
\end{abstract}

\section{Introduction}

Different detector technologies have been developed in the last decades with the aim to directly detect dark matter particles which could be pervading the galactic halo [1]. Looking specifically for low mass Weakly Interacting Massive Particles (WIMPs) requires the use of light elements as target, detectors with very low energy threshold, well below

\footnotetext{
a e-mail: scebrian@unizar.es
}

$1 \mathrm{keV}_{e e},{ }^{1}$ and very low radioactive background. Results from either new implementations of semiconductor detectors with extremely low readout capacitance [2-4] or reoriented experiments focused on low threshold [5-13] have already been presented. Gas Time Projection Chambers (TPCs) equipped with Micromegas planes have excellent features to fulfill these requirements. TREX-DM (TPC for Rare Event eXperiments-Dark Matter) $[14,15]$ is a Micromegasread High Pressure TPC for low mass WIMP searches using Ar or Ne mixtures, not focused on directionality. The detector was built and operated at surface in the University of Zaragoza as proof of concept. The experiment was approved by the Canfranc Underground Laboratory (LSC) in Spain and the whole detector was moved underground in 2018. The data taking is expected to start using $\mathrm{Ne}$ once the commissioning underground along 2019 is completely finished.

The Micromegas are consolidated readout structures; a micro-mesh is suspended over a pixelated anode plane, forming a thin gap where charge amplification takes place. Detectable signals in the anode and the mesh are generated. Different technologies have been built: bulk Micromegas have the readout plane and the mesh all in one and microbulk Micromegas are in addition more homogeneous and radiopure [16]. They offer important advantages for rare event detection $[15,17]$ : the possibility of scaling-up, topological information to discriminate backgrounds from the expected signal (just a few microns track for dark matter particles, giving a point-like event) and low intrinsic radioactivity as they are made out of kapton and copper, potentially very clean.

Operating deep underground at ultra-low background conditions is a must in experiments looking for rare events like the direct detection of WIMPs. In this kind of experiments, the construction of reliable background models, based on an

\footnotetext{
${ }^{1}$ Electron equivalent energy.
} 
accurate assay of background sources and on a careful computation of their contribution to the experiment, is essential; they provide guidance and constraints for design and allow robust estimations of the experiment sensitivity (some recent examples can be found at [18-27]). The preliminary background model of TREX-DM for operation at LSC presented in $[14,28]$ has now been completed and updated, including as inputs the activities from a dedicated material screening program together with the measured fluxes of different backgrounds at LSC (gamma-rays, neutrons and muons).

The structure of the article is the following. The detector set-up and its performance are presented in Sect. 2. Section 3 describes the simulations carried out. Then, the results obtained in the material radioassay campaign are detailed in Sect. 4. The estimates of the contribution of each one of the background components considered are shown in Sect. 5. Finally, the corresponding sensitivity for WIMP direct detection and conclusions are discussed in Sects. 6 and 7.

\section{Experimental set-up}

The TREX-DM detector, as built and operated at the University of Zaragoza, was described in detail in [14]; in the set-up at LSC some modifications have been implemented: operation is made with non-flammable gas mixtures, microbulk (instead of bulk) Micromegas are being used read by a new AGET-based DAQ system and a full detector shielding is in place.

Two active volumes $\left(19 \times 25 \times 25 \mathrm{~cm}^{3}\right.$ each $)$ are separated by a central cathode made of mylar inside a 6 -cm-thick copper vessel, designed and certified as a pressure equipment to hold up to 11 bar(a) or 10 barg (see Fig. 1, middle). The field cage, made of kapton and copper using 42 Finechem resistors, is covered by teflon. Two microbulk Micromegas fabricated at CERN are used; they are the largest area single microbulk readout produced so far, with an active area of $25 \times 25 \mathrm{~cm}^{2}$ (see Fig 1, top). Flat cables take out signals from strips and connect to the interface cards out of the vessel. The connections at both sides of the flat cables are now made through special silicone-based connectors (Zebra Gold 8000C from Fujipoly), checked to be more radiopure than the connectors firstly used (see Sect. 4). Signals from $2 \times 256$ strips (with $\sim 1 \mathrm{~mm}$ pitch) at each side are digitized for tracking in a $10.2 \mu$ s window (512 samples, $50 \mathrm{MHz}$ sampling rate). 3D track reconstruction is possible, using the position of hit strips to determine $\mathrm{X}$ and $\mathrm{Y}$ coordinates, and electron drift time information to get the $\mathrm{Z}$ coordinate. The AGET-based DAQ system consists of four front-end (FECAGET) cards, containing 4 AGET chips each for sampling of the pulses, four mezzanine (FEMINOS) cards, subtracting pedestals, and a trigger module (TCM), connected by Ethernet cables. It provides self-triggered data acquisition

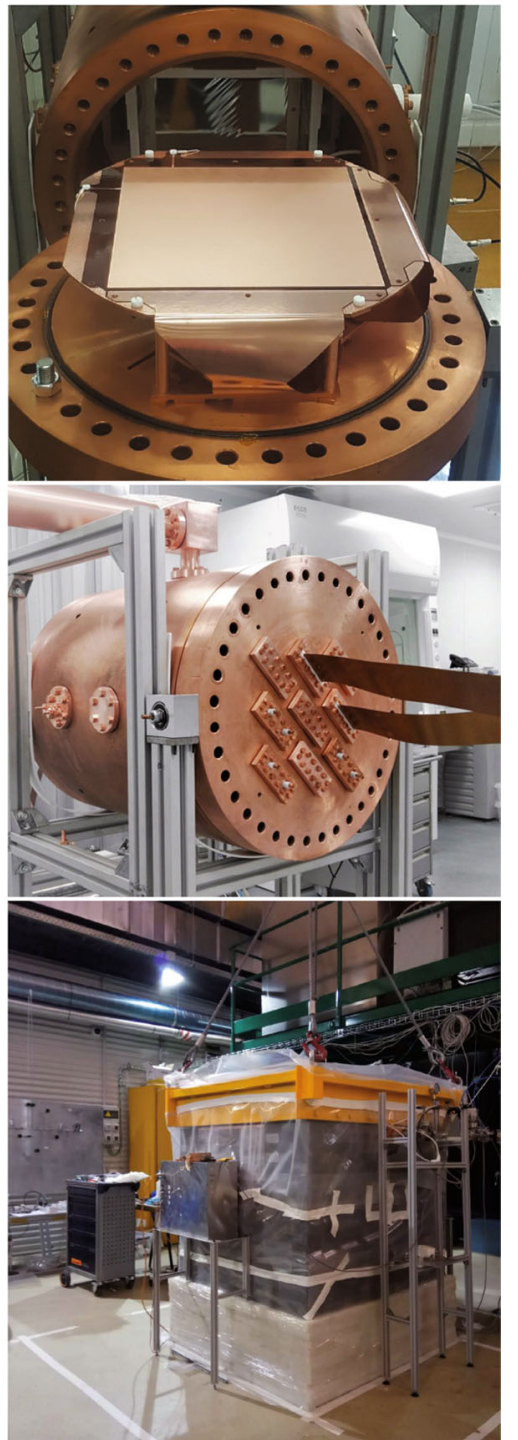

Fig. 1 Pictures of the Microbulk Micromegas of TREX-DM, the largest ever fabricated, being tested (top), the copper vessel at the clean room of LSC during mounting (middle) and the set-up with the lead shielding at hall A of LSC (bottom)

allowing to have a trigger generated individually by each single strip signal; as capacitance of strips is about a factor 30 lower than that of the mesh, the use of AGET electronics was considered in order to improve the energy threshold in comparison to the first operation using AFTER electronics with trigger taken from the mesh signal [14]. Two Faraday cages, one at each end, have been installed housing the interface cards, FEC-AGET and FEMINOS cards (see Fig. 1, bottom).

As shown in Fig. 2, a complete shielding consisting of $5 \mathrm{~cm}$ of copper, $20 \mathrm{~cm}$ of low activity lead and $40 \mathrm{~cm}$ of neutron moderator (water tanks and polyethylene blocks) covers the detector at LSC, having in addition a Rn-free atmosphere inside shielding. The present TREX-DM set-up installed at 

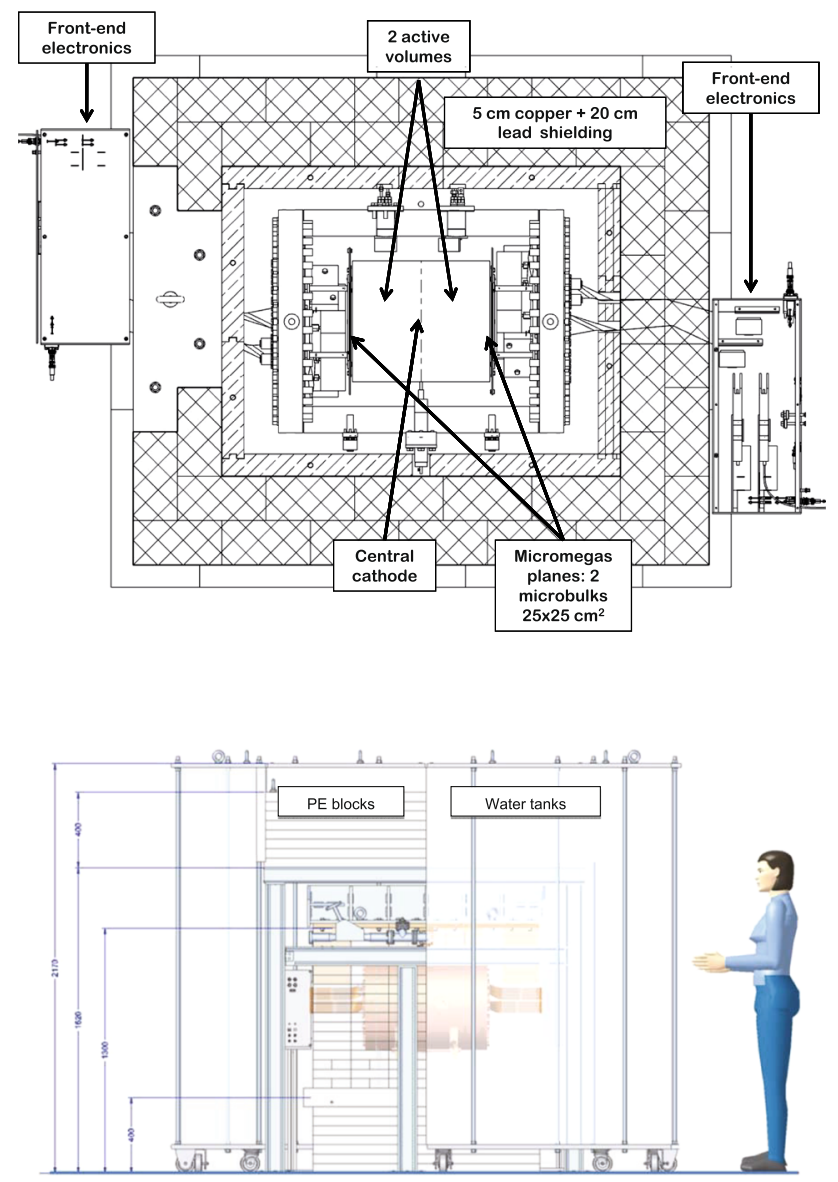

Fig. 2 Top view of the design of the copper and lead shielding (top) and side view of the moderator shielding (bottom) for TREX-DM at LSC

the hall A of the Canfranc laboratory, placed at a depth of 2450 m.w.e., is shown in Fig. 1, bottom, including the lead shielding.

The gas system of TREX-DM has been designed for nonflammable gases, which is accomplished adding $1 \%$ of isobutane to argon and $2 \%$ in the case of neon. This simplified installation underground. It consists of a recirculation part and a purification branch and it can run in two operation modes: in open loop (for commissioning tasks) or in recirculation through a purifier branch (nominal working condition). The gas system installation has been completed and certified by an authorized body to operate at high pressure (up to 11 bar). The slow control system, based on a net of Raspberry PI cards, each of them monitoring or controlling one experimental component (like gas pressure and temperature, voltages or gas flow), is ready and in operation.

First results from the commissioning phase of TREX-DM on surface using bulk Micromegas were shown in [14] for different gas mixtures and pressures. Microbulk Micromegas were characterized afterwards using the mixtures finally considered for the underground operation, $\mathrm{Ar}+1 \% \mathrm{iC}_{4} \mathrm{H}_{10}$ and
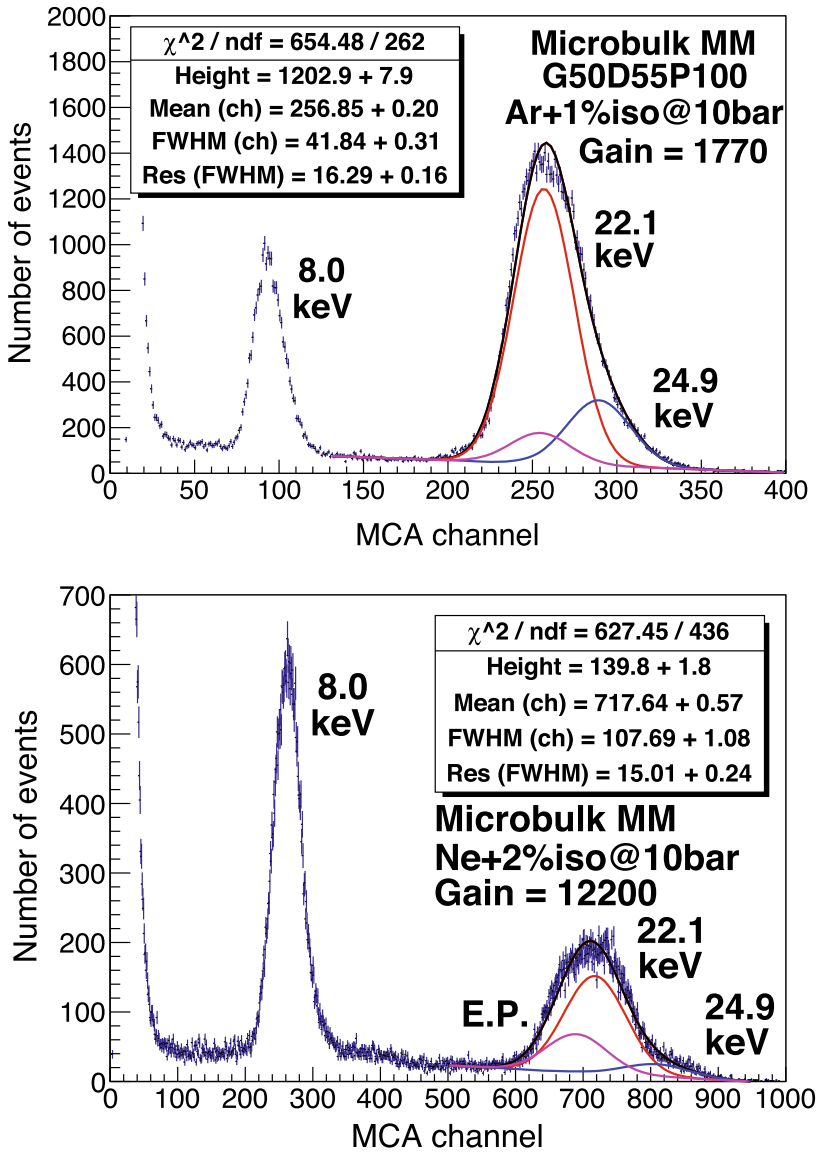

Fig. 3 Energy spectrum obtained in the characterization of microbulk Micromegas with $\mathrm{Ar}$ (top) and $\mathrm{Ne}$ (bottom) mixtures using a ${ }^{109} \mathrm{Cd}$ source at 10 bar. The results of a multi-Gaussian fit for estimating the FWHM at $22.1 \mathrm{keV}$ are shown. The fitted peak is generated by the $\mathrm{K} \alpha$ $(22.1 \mathrm{keV})$ and $\mathrm{K} \beta(24.9 \mathrm{keV}) \mathrm{X}$-ray lines produced by the source, as well as their corresponding escape peaks. The copper K-fluorescence induced by the source at the copper components close to the gas volume is clearly identified at $8 \mathrm{keV}$

$\mathrm{Ne}+2 \% \mathrm{iC}_{4} \mathrm{H}_{10}$, in order to check that the required performance was achieved also in these conditions [29]. These tests were made at $1-10$ bar using a ${ }^{109} \mathrm{Cd}$ source in a smaller setup at the University of Zaragoza (also used in [30]) without shielding. Figure 3 presents the energy spectra registered at 10 bar; energy resolution has shown some degradation with pressure, being the FWHM at 10 bar $16(15) \%$ for $\operatorname{Ar}(\mathrm{Ne})$ at $22.1 \mathrm{keV}$. It must be noted that neither the background level nor the energy threshold in these measurements were representative of those achievable at TREX-DM in LSC. As shown in Fig. 4, an excellent behavior has been registered for gain, with maximum values above $10^{3}\left(10^{4}\right)$ in $\operatorname{Ar}(\mathrm{Ne})$ for all pressures, which is very important for achieving low energy thresholds. In principle, a very low threshold is possible thanks to the intrinsic amplification in gas but the readout area, the sensor capacitance and the electronic noise set in practice the effective threshold in this type of detectors. For 

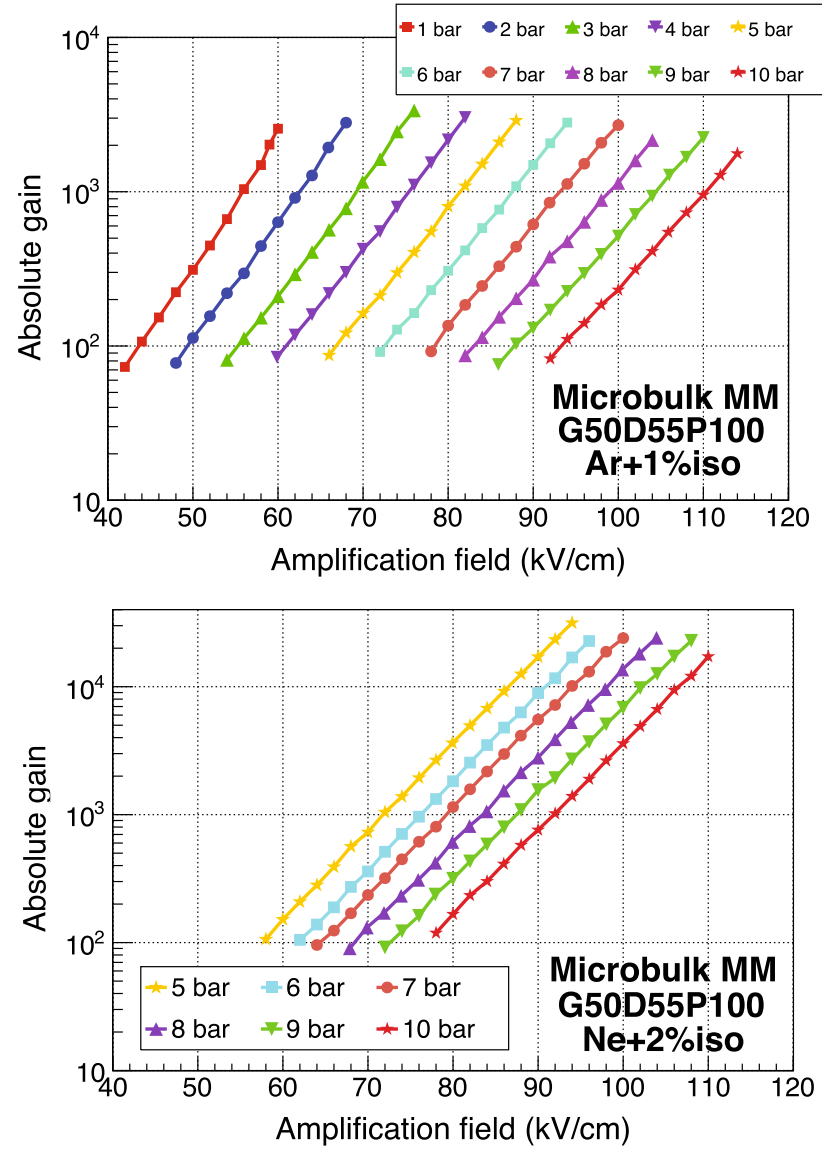

Fig. 4 Gain curves obtained in the characterization of microbulk Micromegas with $\mathrm{Ar}$ (top) and $\mathrm{Ne}$ (bottom) mixtures using a ${ }^{109} \mathrm{Cd}$ source. Curves for different pressures up to 10 bar are shown

TREX-DM, the strip capacitance, including the contributions of the flat cables and the interface cards, is $\sim 0.2 \mathrm{nF}$. The TREX-DM nominal (conservative) aim for effective threshold is $100 \mathrm{eV}_{e e}\left(400 \mathrm{eV}_{e e}\right)$. The very first results from the commissioning data taken in Canfranc also using microbulk Micromegas with atmospheric Ar and with Ne confirm that this expectation is at reach.

At the end of 2018, the detector was already fully equipped (as described in this section) and installed inside its copper and lead castle at LSC. For the first commissioning data, the chamber was filled with atmospheric $\mathrm{Ar}+1 \% \mathrm{iC}_{4} \mathrm{H}_{10}$ and operated at 1-1.5 bar. Calibration measurements made with a ${ }^{109} \mathrm{Cd}$ source indicated that the detector operates as expected in terms of gain and spectral and spatial distribution of events. Few-days long background runs with the expected performance were carried out, being the measured spectra dominated by ${ }^{39} \mathrm{Ar}$. In the beginning of 2019, the gas changed from the test option to the baseline mixture $\mathrm{Ne}+2 \% \mathrm{iC}_{4} \mathrm{H}_{10}$ in recirculation and operation at higher pressures is underway. Data analysis is ongoing to produce quantitative statements on e.g. threshold level and background level. A detailed
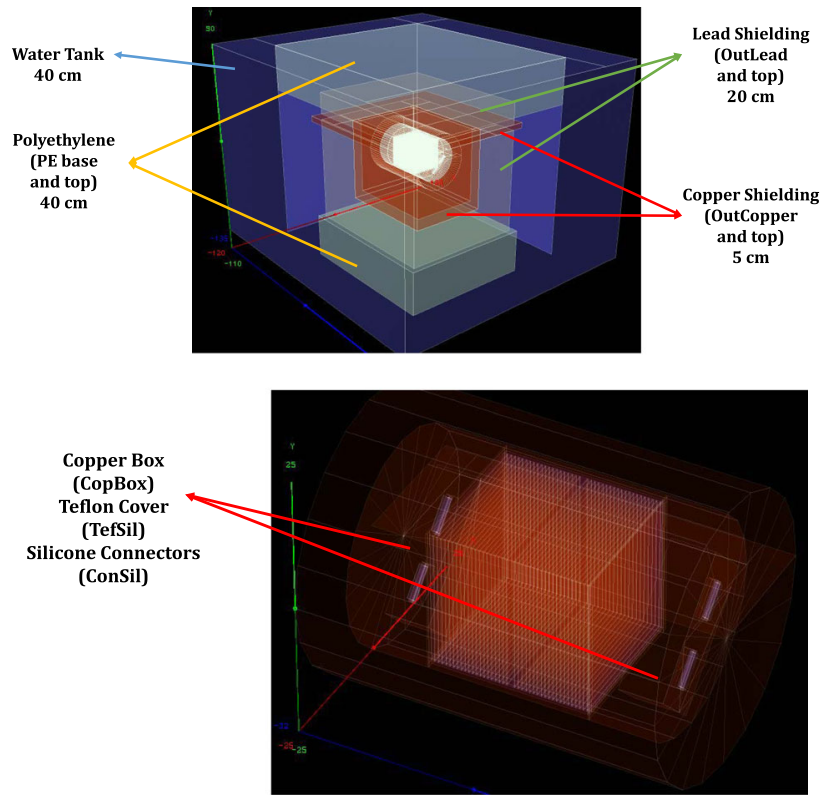

Fig. 5 Views of the geometry of TREX-DM implemented in the GEANT4 simulation code, showing the whole shielding (top) and the copper vessel (bottom)

description of the performance of the detector will be presented in a dedicated technical publication.

\section{Simulation}

The complete simulation of the detector response to build the TREX-DM background model is based on RestG4, a package integrating Geant4 [31] and a custom-made code called REST [32], also used in [33]. Geant 4 version 4.10 has been considered. The Geant4 Radioactive Decay Module is used for the event generation. The low energy models based on Livermore data libraries have been implemented for interactions of alpha, beta and gamma particles. These models are accurate for energies between $250 \mathrm{eV}$ and $100 \mathrm{GeV}$ and can be applied down to $100 \mathrm{eV}$ with a reduced accuracy [34]. The NeutronHP model has been used for neutrons. A detailed geometry of the set-up has been implemented based on the Geometry Description Markup Language (GDML) format (see Fig. 5), including the whole shielding (with copper, lead and neutron moderator) and details of the copper vessel, field cage, cathode, cables and connectors and Micromegas readouts. The ROOT-based code simulates also the electron generation in gas, diffusion effects, charge amplification at Micromegas and signal generation. The resulting data from the simulation chain have the same REST format as the DAQ data in order to share analysis tools, including those for discriminating point-like events from complex topologies.

Simulations to build the TREX-DM background model have been run for the two gas mixtures $\left(\mathrm{Ar}+1 \% \mathrm{iC}_{4} \mathrm{H}_{10}\right.$ and 
$\left.\mathrm{Ne}+2 \% \mathrm{iC}_{4} \mathrm{H}_{10}\right)$ at 10 bar, corresponding to a total active mass of $0.30 \mathrm{~kg}$ for Ar and $0.16 \mathrm{~kg}$ for Ne. A successful validation of the simulation environment against experimental data from calibration measurements has been made [14]; in particular, the shape of the energy spectra and the distribution of the analysis observables measured were checked to be properly reproduced by simulation when irradiating the active volume with a ${ }^{109} \mathrm{Cd}$ source. The event-by-event signal identification and background rejection applied is based on the analysis of the event topology. Firstly, just one-cluster events are selected. This selection criterion hardly reduces the rate a $0.1 \%$ since in the region of interest almost all the events are single-site. The second condition is to be farther than $2 \mathrm{~cm}$ from the lateral faces of the prism defined by the active volume; i.e., a planar fiducial cut. Simulations show an accumulation of events near the border of the readout plane due to photons produced in interactions in surrounding materials (field cage, cathode frame, ...), which disappears after imposing this 2-cm-veto-area. Reduction factors in this case depend very much on the background sources, but have mean values of $\sim 40 \%$. As our detector lies on one of its sides, this veto also eliminates muons traversing directly the detector. The active mass of the detector to obtain the counting rates per mass unit is that of the fiducial volume. So far, the analysis has been quite simple and more complex discrimination algorithms, based on the analysis of current calibration runs, could help to further decrease background levels.

\section{Measurements of material radiopurity}

An exhaustive material radioassay campaign for TREX-DM was undertaken a few years ago [14,17,35], as made in other experiments in the context of rare event searches (see for instance recent results in [36-42]). It has allowed, on one side, to design and construct the detector and shielding according to the radiopurity specifications and, on the other, to provide inputs to build the experiment background model. The material screening program is mainly based on germanium gamma-ray spectrometry carried out deep underground in Canfranc, but complemented by other techniques like GDMS and ICPMS and by measurements using the BiPo-3 detector at LSC. In this section, the techniques employed for the radiopurity measurements are summarized and the relevant results shown and discussed.

\subsection{Techniques}

Most of the germanium measurements have been made using $\mathrm{a} \sim 1 \mathrm{~kg}$ ultra-low background detector of the University of Zaragoza (named Paquito) operated at the hall E of the LSC. This detector has been used for radiopurity measurements at
Canfranc for many years (details on the features and performance of the detector can be found in $[35,43])$. Some of the $\sim 2 \mathrm{~kg}$ close-end coaxial HPGe detectors of the Radiopurity Service of LSC [44] have been used for some measurements too. Activities of different sub-series in the natural chains of ${ }^{238} \mathrm{U},{ }^{232} \mathrm{Th}$ and ${ }^{235} \mathrm{U}$ as well as of common primordial, cosmogenic or anthropogenic radionuclides like ${ }^{40} \mathrm{~K},{ }^{60} \mathrm{Co}$ and ${ }^{137} \mathrm{Cs}$ are typically evaluated. The detection efficiency is determined by Monte Carlo simulations based on Geant 4 for each sample, validated with a ${ }^{152} \mathrm{Eu}$ reference source [35]; a conservative overall uncertainty on the deduced efficiency is properly propagated to the final results.

Glow Discharge Mass Spectrometry (GDMS) has been performed by Evans Analytical Group in France, providing concentrations of $\mathrm{U}$, Th and $\mathrm{K}$. In addition, thanks to the collaboration of LSC, Inductively Coupled Plasma Mass Spectrometry (ICPMS) analysis carried out at the Laboratori Nazionali del Gran Sasso (LNGS) [45] has been possible for some TREX-DM samples quantifying the $U$ and Th concentrations. It must be noted that when having no information on daughter radionuclides in the chains, a possible disequilibrium cannot be detected.

Taking advantage of the "foil" geometry of some samples, very sensitive measurements have been made in the BiPo-3 detector [46], in operation at LSC. This detector has been developed by the SuperNEMO collaboration and is able to measure extremely low levels, down to a few $\mu \mathrm{Bq} / \mathrm{kg}$, of ${ }^{208} \mathrm{Tl}$ and ${ }^{214} \mathrm{Bi}$ radioactivity in very thin samples (below $200 \mu \mathrm{m}$ thick) by registering the delayed coincidence between electrons and alpha particles occurring in the BiPo events. These measurements can be translated into contamination of natural $\mathrm{U}$ and $\mathrm{Th}$ chains if secular equilibrium is assumed.

\subsection{Results}

A large amount of materials and components related to Micromegas readout planes and the whole set-up (the gas vessel, the field cage, the radiation shielding or the electronic acquisition system) has been taken into consideration in the screening program. Massive elements and those in contact with the sensitive volume of the detector are in principle the most relevant. Table 1 reproduces, for the sake of completeness, the previous activity results obtained for the components finally used in the TREX-DM set-up and presents all the new ones; reported errors include both statistical and efficiency uncertainties.

\subsubsection{Shielding and vessel}

Many samples from different suppliers of lead, used for shielding, and copper, used for mechanical and electric components (like Micromegas plates, cathodes, HV feedthroughs 
or field cage rings), have been analyzed [14]. Results are available for the lead bricks used in the shielding, provided by Mifer (\#1 of Table 1) and by LSC (from lead coming from the OPERA experiment [47]). Very stringent upper limits were also obtained by GDMS for Oxygen Free Electronic (OFE, C10100) copper from the Luvata company and the used Electrolytic Tough Pitch (ETP, C11000) copper from Sanmetal was measured too (\#2-3 of Table 1).

A sample of the copper provided by Sanmetal and used at the vessel of TREX-DM, having the same origin and history of exposure to cosmic rays on surface as the vessel itself, has been additionally screened using one of the germanium detectors of the LSC Radiopurity Service with the aim to evaluate the cosmogenic activation induced in the vessel and then to assess its suitability for a low background measurement at LSC. The measured ${ }^{60} \mathrm{Co}$ activity (\#4 of Table 1) is in very good agreement with expectations for production rates of $\sim 50 \mathrm{~kg}^{-1} \mathrm{~d}^{-1}=0.58 \mathrm{mBq} / \mathrm{kg}$ (corresponding to the order of different direct measurements and calculations from the literature [48]) and an exposure time of $\tau / 2=3.80 \mathrm{y}$. The activity of other isotopes with half-lives shorter than ${ }^{60} \mathrm{Co}$ has also been evaluated: $(0.35 \pm 0.07) \mathrm{mBq} / \mathrm{kg}$ of ${ }^{58} \mathrm{Co},<0.81 \mathrm{mBq} / \mathrm{kg}$ of ${ }^{57} \mathrm{Co}$ and $<0.29 \mathrm{mBq} / \mathrm{kg}$ of ${ }^{54} \mathrm{Mn}$. Thanks to the low energy threshold of the GeOroel detector, it was possible in this measurement to observe the $46.5 \mathrm{keV}$ gamma line from ${ }^{210} \mathrm{~Pb}$ and to derive an upper limit on its activity (considering the efficiency for surface emissions) as $<0.32 \mathrm{mBq} / \mathrm{cm}^{2}$.

Two samples of tube intended to be used at the calibration system of TREX-DM, traversing shielding and vessel have been screened (\#5-6 of Table 1). One was made of PFA (PerFluoroAlkoxy polymer) produced by Emtecnik and the other was made of PTFE supplied by Tecnyfluor. Upper limits have been set for all the common radioisotopes for both tubes and therefore any of them can be used.

\subsubsection{Field cage}

Material and components to be used inside the gas vessel, mainly related to the field cage, were screened to select proper teflon, resistors or adhesives.

The monolayer Printed Circuit Board (PCB) made of kapton and copper for the field cage, supplied by LabCircuits, was found to have good radiopurity [14], but since the upper limits set on the activity were too high for the required sensitivity, a new sample with larger surface was measured afterwards (\#7 of Table 1), providing a reduction of at least a factor 10 in the relevant isotopes.

For the epoxy resin Hysol RE2039 from Henkel no contaminant could be quantified $[14,35]$ (\#8 of Table 1 ) and it is being used for gluing.

Surface Mount Device (SMD) resistors supplied by Finechem showed lower activity than other equivalent units
$[14,35]$ (\#9 of Table 1) and therefore were chosen for the field cage.

A sample of the 3.5- $\mu$ m-thick mylar sheet from Goodfellow used at the cathode of TREX-DM has been screened (\#10 of Table 1), setting upper limits for all the common radiosotopes.

The possibility of using 3D printing to produce easily some of the mechanical components of the field cage of TREX-DM, instead of fabricating them using commercial teflon, was considered and the possible impact on background evaluated. A sample made of PA2200 2 produced by a 3D printer at the Centro Nacional de Microelectronica (CNM), Barcelona, was first screened using a germanium detector (\#11 of Table 1) setting upper limits for all the usual radiosotopes. In an attempt to reduce the available limits on activity for several plastic samples, which would give a non-negligible contribution to the TREX-DM background model, an ICPMS analysis was carried out at LNGS for three samples. For all of them the mineralization was performed using the dry ashing technique and the uncertainty of the measurement can be estimated as 30\% of the given values. For teflon from Sanmetal, the ICPMS limits set for the activity of ${ }^{232} \mathrm{Th}$ and ${ }^{238} \mathrm{U}$ chains (\#13 of Table 1) improve by about two orders of magnitude those obtained using the Paquito detector at LSC $[14,35]$. Another type of teflon, extruded PTFE (Gore GR gasketing), was also analyzed, pointing to a similar radiopurity (\#14 of Table 1). For a small piece of the nylon PA2200 from 3D printing, previously screened at a germanium detector setting upper limits for all common radioisotopes, both $\mathrm{U}$ and $\mathrm{Th}$ concentrations could be quantified. As shown in \#12 of Table 1, the corresponding ${ }^{232} \mathrm{Th}$ activity is compatible with the upper limits set using germanium detectors, but being of the same order. For ${ }^{238} \mathrm{U}$, the activity value from ICPMS is compatible with that for the upper part of the chain from germanium (the actual comparable result between both techniques) but higher that than for the lower part. Therefore, following these radiopurity results and given the important contribution expected from a nylon PA2200 field cage in the background model, its use has been disregarded and teflon has been employed instead.

\subsubsection{Electronics}

Electronic connectors made of Liquid Crystal Polymer (LCP) have shown unacceptable activities of at least several $\mathrm{mBq} / \mathrm{pc}$ for isotopes in ${ }^{232} \mathrm{Th}$ and the lower part of ${ }^{238} \mathrm{U}$ chains and for ${ }^{40} \mathrm{~K}[14,35,39,40]$. Three different types of siliconebased connectors supplied by Fujipoly have been screened: Gold 8000 connectors type C, units from Zebra Series 5002 SZ100 made of silver, and connectors from Zebra Series 2004

\footnotetext{
${ }^{2}$ PolyAmide white or polyamide 12 or nylon 12 .
} 


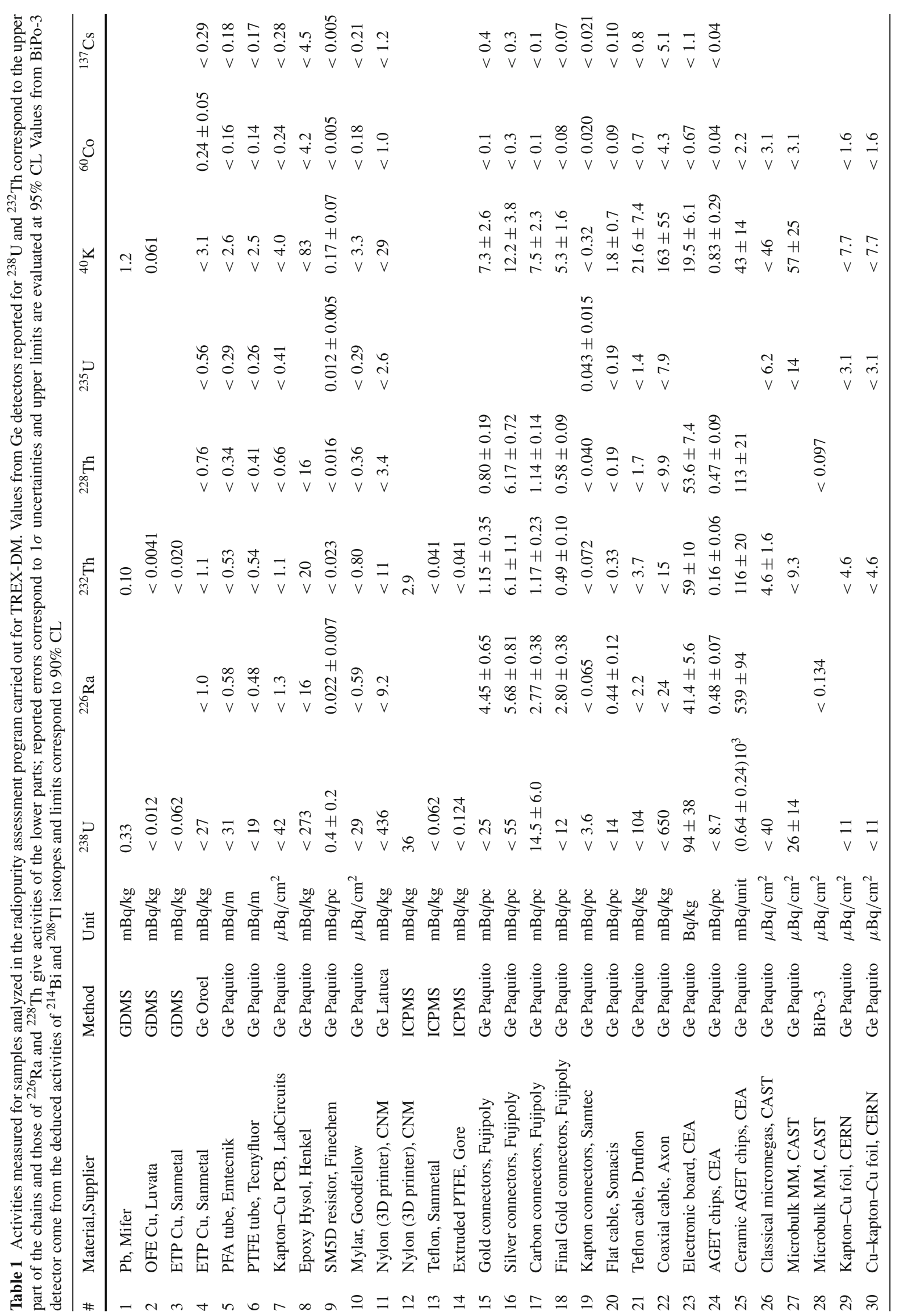




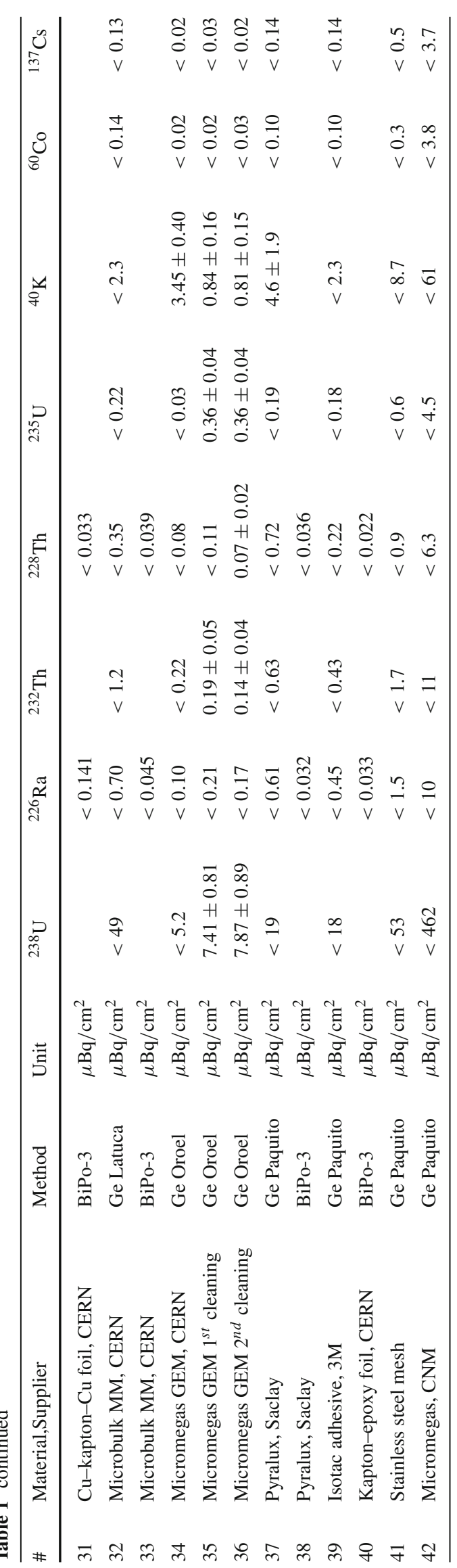


CZ418 made of carbon (\#15-17 of Table 1); although activities of ${ }^{40} \mathrm{~K},{ }^{232} \mathrm{Th}$ and the lower part of the ${ }^{238} \mathrm{U}$ chain have been quantified in all cases, much lower values than in LCP connectors have been obtained. For silver connectors, activity from ${ }^{208 m} \mathrm{Ag}\left(\mathrm{T}_{1 / 2}=428 \mathrm{y},(0.38 \pm 0.05) \mathrm{mBq} / \mathrm{pc}\right)$ and ${ }^{210 m} \mathrm{Ag}\left(\mathrm{T}_{1 / 2}=249.78 \mathrm{~d},(1.88 \pm 0.12) \mathrm{mBq} / \mathrm{pc}\right)$ has been assessed too. Due to these results, Gold Zebra 8000C connectors were selected for TREX-DM; a new screening of the units to be actually used has been made (\#18 of Table 1) confirming that in comparison with the Samtec LCP connectors firstly used in the set-up at the University of Zaragoza [35], the silicone ones of the present set-up have about a factor 32 less activity of ${ }^{232} \mathrm{Th}$ and reduction is about 3.3 for ${ }^{226} \mathrm{Ra}$ and 2.3 for ${ }^{40} \mathrm{~K}$. Recently, very promising results have been found in the screening of new connectors produced by Samtec (Ref. ZA8H-24-0.33-Z-07) (\#19 of Table 1), as only upper limits have been set for all the common radioisotopes, being more than one order of magnitude lower than the quantified activities in the Fujipoly ones. Comparing to the firstly used Samtec connectors, the reduction is around two orders of magnitude. This has been possible due to the very reduced mass of each unit $(0.087 \mathrm{~g} / \mathrm{unit})$ and the use of only kapton and copper as base materials. This is an important result to take into consideration in future upgrades of the detector.

Very radiopure, flexible, flat cables made of kapton and copper have been developed in collaboration with Somacis, performing a careful selection of the materials included and avoiding glass fiber-reinforced materials at base plates. After the screening of several cable designs [14], the good results obtained for the final one (\#20 of Table 1) allow to envisage the use of these materials also at Micromegas production. Several kinds of high voltage or signal cables have been analyzed too [14]. Screened cables from Druflon Electronics (to connect the field cage last ring to HV feedthrough) and coaxial low noise cable from Axon Cable S.A.S. (to extract the mesh signal from the vessel) are being used. Only ${ }^{40} \mathrm{~K}$ activity was quantified for the two cables (\#21-22 of Table 1), made basically of copper and teflon, showing much better radiopurity than typical RG58 coaxial cables.

Different materials can be taken into consideration for PCBs and samples of FR4, ceramic-filled PTFE composite and cuflon were screened [14]. The first ones presented very high activities for the natural chains and ${ }^{40} \mathrm{~K}$, precluding its use. Good radiopurity was found for cuflon from Crane Polyflon; however, its application for Micromegas has been disregarded due to the difficulty to fix the mesh and also because bonding films to prepare multilayer PCBs have been shown to have unacceptable activity [40]. Following the measurements \#7 and \#20 of Table 1, kapton-copper boards seem to be the best option.

One of the electronic boards used at TREX-DM (with approximate surface $14 \times 25 \mathrm{~cm}^{2}$ ) has been directly screened
(\#23 of Table 1). Values of specific activity obtained for ${ }^{232} \mathrm{Th}$ and ${ }^{238} \mathrm{U}$ chains are a factor $2-3$ larger than those measured in a raw PCB made mainly of FR4 from Somacis company [14], which seems to point out to an additional relevant source of activity in the electronic components of the board. A sample of non-functional AGET chips $(2.74 \mathrm{~g} / \mathrm{pc})$ provided by CEA Saclay has been screened too. Following the results in \#24 of Table 1, each chip has a few tenths of $\mathrm{mBq}$ of ${ }^{40} \mathrm{~K}$ and of the isotopes of the ${ }^{232} \mathrm{Th}$ and ${ }^{238} \mathrm{U}$ chains. These nonnegligible quantified activities in the electronic components should not pose a problem since they are located outside the copper and lead shielding of the DAQ system. As part of the common effort to develop radiopure electronic components in collaboration with CEA Saclay, a sample of four units of AGET chips with a different ceramic cover provided by CEA was analyzed too (\#25 of Table 1). Specific activities of the order of tens of $\mathrm{Bq} / \mathrm{kg}$ have been measured for the isotopes of the ${ }^{232} \mathrm{Th}$ and ${ }^{238} \mathrm{U}$ chains; the corresponding activities per unit are from two to three orders of magnitude higher in comparison to those previously obtained for AGET chips with plastic cover. Therefore, the use of this type of ceramic packaging must be avoided. A large sample of naked chips has been screened too at the Modane Laboratory confirming a very good radiopurity.

\subsubsection{Micromegas}

The radiopurity of Micromegas readout planes was first analyzed in depth in [43]. On the one hand, two samples (\#2627 of Table 1) were part of fully functional Micromegas detectors: a full microbulk readout plane formerly used in the CAST experiment and a classical Micromegas structure without mesh. On the other hand, two other samples (\#2930 of Table 1) were screened corresponding just to the raw foils used in the fabrication of microbulk readouts, consisting of kapton metalized with copper on one or both sides. The raw materials (kapton and copper, mainly) were confirmed to be very radiopure; the numbers for the treated foils show similar limits or values just at the limit of the sensitivity of the germanium measurement. Despite their importance, these bounds were still relatively modest when expressed in volumetric terms, due to the small mass of the samples. New activity measurements for the microbulk Micromegas and $\mathrm{Cu}-\mathrm{kapton}-\mathrm{Cu}$ foil samples (previously measured with $\mathrm{Ge}$ spectroscopy) were carried out profiting the great capabilities of the BiPo-3 detector operating at LSC (\#28,31 of Table 1). For both cases, only limits to the contamination in ${ }^{208} \mathrm{Tl}$ and ${ }^{214} \mathrm{Bi}$ can be deduced, which improve the Ge spectrometry limits by more than 2 orders of magnitude, pointing to contaminations at the level of, or below, $\sim 0.1 \mu \mathrm{Bq} / \mathrm{cm}^{2}$ [17]. A more sensitive measurement for microbulk Micromegas produced at CERN was made in 2016, using two capsules of the BiPo-3 detector $\left(30 \times 30 \mathrm{~cm}^{2}\right.$ each $)$; results shown 
in \#33 of Table 1 point to a very significant reduction of the upper limits of both ${ }^{208} \mathrm{Tl}$ and ${ }^{214} \mathrm{Bi}$. All this confirms our expectations that microbulk readouts contain radioactivity levels well below typical components in very low background detectors.

As the BiPo-3 detector can only quantify activity from the $\mathrm{U}$ and Th chains, but a non-negligible $\mathrm{K}$ content seemed to be present in the first analysis of microbulk Micromegas [43], a new, more sensitive analysis of the ${ }^{40} \mathrm{~K}$ activity was undertaken since its contribution in the background model was found to be important [14]. A sample of the same microbulk Micromegas analyzed in the BiPo-3 detector was screened using a germanium detector (\#32 of Table 1) deriving upper limits for all the common radioisotopes. Limits set for ${ }^{238} \mathrm{U}$ and ${ }^{232} \mathrm{Th}$ chains are higher than those derived from BiPo-3 measurement as expected. The limit for ${ }^{40} \mathrm{~K}$ is $<2.3 \mu \mathrm{Bq} / \mathrm{cm}^{2}$, a factor 25 lower than the estimated value in [43]; even if very promising, it was not conclusive yet as the analyzed sample had not the holes produced by the potassium compound which could be responsible of a ${ }^{40} \mathrm{~K}$ contamination. Then, a new, more massive sample of readouts from CERN with a total surface of $12,372.75 \mathrm{~cm}^{2}$ was analyzed. It consisted of faulty GEMs glued on kapton, produced as microbulk Micromegas, which have gone through some chemical baths involving potassium compounds to create the kapton holes. This sample has been screened up to three times:

- In the first screening in a germanium detector, as shown in \#34 of Table 1, upper limits have been set for all the common radioisotopes except for ${ }^{40} \mathrm{~K}$; those of ${ }^{232} \mathrm{Th}$ and ${ }^{238} \mathrm{U}$ activity are, as expected, higher than the ones obtained from the BiPo-3 detector (about a factor of 5.7 and 2 , respectively). The signal from ${ }^{40} \mathrm{~K}$ is clear and its activity has been quantified as (3.45 \pm $0.40) \mu \mathrm{Bq} / \mathrm{cm}^{2}$ (corresponding to a specific activity of $(258 \pm 30) \mathrm{mBq} / \mathrm{kg})$. This seemed to confirm that the potassium content is related to the production of holes in the readout.

- In an attempt to reduce potassium, the same sample with holes was cleaned in water baths at CERN (dipped 1 week in tap water followed by a long rinse with deionized (DI) water) and a new screening to assess the effect of this treatment using the same detector was made. As presented in \#35 of Table 1 , the measured ${ }^{40} \mathrm{~K}$ activity is $(0.84 \pm 0.16) \mu \mathrm{Bq} / \mathrm{cm}^{2}$, reduced by a factor 4 , but an important uranium activity is unexpectedly obtained. The ratio of activities of the mothers of the ${ }^{238} \mathrm{U}$ and ${ }^{235} \mathrm{U}$ chains is 20.6 , in very good agreement with the expectation for natural uranium. The origin of the relevant uranium contamination found, at the level of $(564 \pm 62) \mathrm{mBq} / \mathrm{kg}$ of ${ }^{238}$ or $(45.8 \pm 5.0) \mathrm{ppb}$ of $\mathrm{U}$, is unknown. A possibility is that it is related to the tap water used for baths ${ }^{3}$. Presence of ${ }^{7} \mathrm{Be}\left(\mathrm{T}_{1 / 2}=53.22\right.$ days, decaying by Electron Capture to ground or excited states) has been identified too thanks to the $477.6 \mathrm{keV}$ gamma emission; due to its half-life, comparable to the measuring time, no direct estimate of the activity has been attempted.

- A second cleaning was made in the same sample in order to assess its effect on both the uranium and the potassium content quantified. In this case the baths were performed only with DI water (for 1 week the sample was cleaned with DI water, changed each day and heated to $60^{\circ} \mathrm{C}$ ). The obtained results (\#36 of Table 1), with no significant change in the derived activities, point to a null effect of the new procedure for both the ${ }^{40} \mathrm{~K}$ and $\mathrm{U}$ activities.

Following these results, alternative cleaning procedures and even the possibility of etching kapton by plasma, totally avoiding potassium compounds, are being studied. It is also worth noting that no indication of a possible ${ }^{210} \mathrm{~Pb}$ contamination has been found in any of the measurements for this sample, as there is no excess over background at the $46.5 \mathrm{keV}$ peak.

A number of other samples involved in various Micromegas fabrication processes have also been measured [17]. A sample of pyralux sheets, used in the construction of bulk Micromegas, showed good radiopurity first in germanium screening (\#37 of Table 1) and afterwards using the BiPo3 detector (\#38 of Table 1). This result is of interest for the development of radiopure bulk Micromegas. A kapton-epoxy foil (AKAFLEX, from Krempel GmbH) used in the microbulk fabrication process (to join several kapton layers in more complex routing designs) has been measured in BiPo-3 (\#40 of Table 1) showing similar values to the previous samples. In addition, a sample of adhesive Isotac 3M VHB used in Micromegas readouts has been screened with a germanium detector (\#39 of Table 1) setting upper limits for all the common radioisotopes. A sample of the stainless steel mesh used in the bulk Micromegas firstly used in TREX-DM (produced by Somacis) has been screened (\#41 of Table 1) deriving only upper limits for all the analyzed radioisotopes.

Alternative production procedures for Micromegas are being explored. Two units of Micromegas (diameter $10 \mathrm{~cm}$, thickness $500 \mu \mathrm{m}$, made of Si covered by silicon oxide SU8 and aluminum) produced at the Centro Nacional de Microelectronica, Barcelona, have been screened (\#42 of Table 1). Upper limits have been set for all the common radioisotopes, pointing to a radiopure production process at CNM in this first approach.

\footnotetext{
${ }^{3}$ Levels of uranium in drinking water are of the order of $10 \mu \mathrm{g} / 1$ or $10 \mathrm{ppb}$, being possible a large dispersion around this value.
} 
Table 2 Background rates (in counts $\mathrm{keV}^{-1} \mathrm{~kg}^{-1} \mathrm{~d}^{-1}$ ) expected in the $\mathrm{RoI}\left(0.2-7 \mathrm{keV}_{e e}\right)$ from activity in components and backgrounds at LSC using Ar or Ne mixtures in TREX-DM. The numbers with \# at the second column refer to Table 1, indicating the activity values considered.
Two total rates are presented for internal and external components: one calculated only from the quantified sources and another where all the contributions including upper limits have been taken into account

\begin{tabular}{|c|c|c|c|}
\hline Component & Reference & Argon & Neon \\
\hline Vessel (primordial) & $\# 2$ & $<0.095$ & $<0.114$ \\
\hline Vessel (cosmogenic) & $\# 4$ & 1.31 & 1.60 \\
\hline Copper Boxes (primordial) & $\# 2$ & $<0.020$ & $<0.020$ \\
\hline Copper Boxes (cosmogenic) & see Sect. 5.2.1 & 0.026 & 0.029 \\
\hline Field Cage (PTFE) & $\# 13$ & $<0.036$ & $<0.052$ \\
\hline Field Cage (resistors) & $\# 9$ & $<0.34$ & $<0.48$ \\
\hline Field Cage (kapton-Cu PCB) & $\# 7$ & $<1.12$ & $<1.54$ \\
\hline Field Cage (cable) & $\# 20$ & $<0.027$ & $<0.037$ \\
\hline Cathode (copper) & $\# 2$ & $<3 \times 10^{-6}$ & $<4 \times 10^{-6}$ \\
\hline Cathode (PTFE) & $\# 13$ & $<1.0 \times 10^{-4}$ & $<1.4 \times 10^{-4}$ \\
\hline Flat Cables & $\# 19$ & $<0.0071$ & $<0.0084$ \\
\hline Connectors & $\# 18$ & 0.083 & 0.022 \\
\hline Epoxy & $\# 8$ & $<0.0028$ & $<0.00094$ \\
\hline Mesh Cable & $\# 21$ & $<3.4 \times 10^{-4}$ & $<1.8 \times 10^{-4}$ \\
\hline Other PTFE Components & $\# 13$ & $<0.019$ & $<0.027$ \\
\hline Readout planes & $\# 30,37$ & $<2.30$ & $<2.68$ \\
\hline Argon gas $\left({ }^{39} \mathrm{Ar}\right)$ & {$[56]$} & 0.16 & \\
\hline Argon gas $\left({ }^{85} \mathrm{Kr}\right)$ & {$[56]$} & 0.52 & \\
\hline Total from internal components & & $<6.1$ & $<6.6$ \\
\hline Total from quantified internal activity & & $4.25 \pm 0.36$ & $4.20 \pm 0.43$ \\
\hline Cosmogenic ${ }^{60} \mathrm{Co}$ in $\mathrm{Cu}$ shielding & see Sect. 5.2.1 & $0.0250 \pm 0.0018$ & $0.0288 \pm 0.0020$ \\
\hline${ }^{222} \mathrm{Rn}$ in air & [44] & $0.1495 \pm 0.0024$ & $0.0841 \pm 0.0013$ \\
\hline Surface ${ }^{210} \mathrm{~Pb}$ on $\mathrm{Cu}$ vessel & see Sect. 5.3 & $<3.5 \times 10^{-3}$ & $<6.2 \times 10^{-3}$ \\
\hline Surface ${ }^{210} \mathrm{~Pb}$ on $\mathrm{Cu}$ shielding & see Sect. 5.3 & $<0.025$ & $<0.034$ \\
\hline Muons (+ muon-induced neutrons) & [69] & $0.205 \pm 0.021$ & $0.336 \pm 0.034$ \\
\hline Neutrons at LSC & {$[71]$} & $(2.52 \pm 0.22) \times 10^{-2}$ & $(7.06 \pm 0.61) \times 10^{-2}$ \\
\hline Neutrons from ${ }^{238} \mathrm{U}$ fission in $\mathrm{Pb}$ & see Sect. 5.6 & $(5.82 \pm 0.39) \times 10^{-5}$ & $(1.094 \pm 0.074) \times 10^{-4}$ \\
\hline Neutrons from ${ }^{238} \mathrm{U}$ fission in $\mathrm{Cu}$ & see Sect. 5.6 & $<2.1 \times 10^{-6}$ & $<4.1 \times 10^{-6}$ \\
\hline Radiogenic neutrons in $\mathrm{Cu}, \mathrm{PTFE}$, steel and polyethylene & Table 5 & $<5.610^{-4}$ & $<1.110^{-3}$ \\
\hline Total from external components & & $<0.43$ & $<0.56$ \\
\hline Total from quantified sources & & $0.40 \pm 0.02$ & $0.52 \pm 0.03$ \\
\hline
\end{tabular}

\section{Background contributions}

The main background sources have been simulated to evaluate their contribution to the counting rate in the region of interest for dark matter searches: radioactive isotopes in the elements of the set-up, either primordial or cosmogenically produced; radon-induced activity; and backgrounds at the LSC including gamma-rays, muons and neutrons. Concerning the measured activities, whenever different values were obtained for the upper and lower parts of the ${ }^{238} \mathrm{U}$ and ${ }^{232} \mathrm{Th}$ chains (see Table 1), they were properly considered in the corresponding simulations. The background levels quoted in the following are referred to a Region of Interest (RoI) of $0.2-7 \mathrm{keV}_{e e}$; the upper value is fixed to avoid the interference from the copper fluorescence peak at $8.0 \mathrm{keV}$ while the low energy threshold assumed is equivalent to $1 \mathrm{keV}$ for $\mathrm{Ar}$ and Ne nuclear recoils. As made in [14], since the quenching factor of neither gaseous argon nor neon has been measured yet, its parametrization in terms of the atom number and mass number from [49] has been considered here. Results for all the components included in the model and the different backgrounds at the laboratory are discussed in 

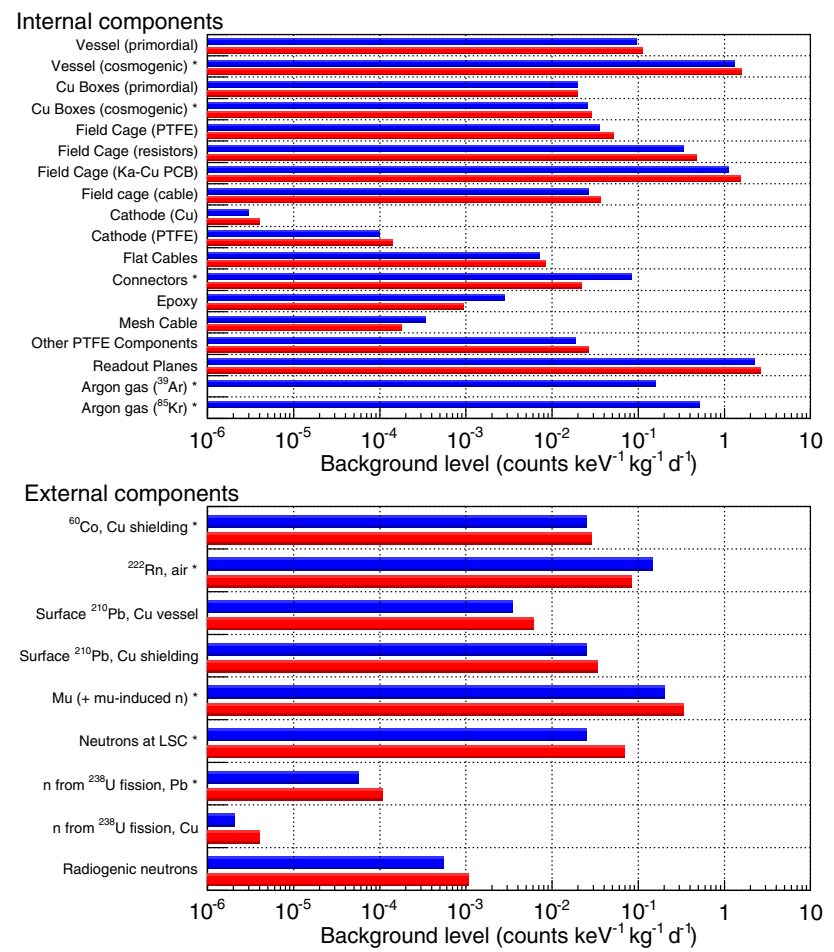

Fig. 6 Background rates (in counts $\mathrm{keV}^{-1} \mathrm{~kg}^{-1} \mathrm{~d}^{-1}$ ) expected in the $\mathrm{RoI}\left(0.2-7 \mathrm{keV}_{e e}\right)$ using $\mathrm{Ar}$ (blue bars) or $\mathrm{Ne}$ (red bars) mixtures in TREX-DM, as shown in Table 2. Rates from contributions inside (top) and outside (bottom) the vessel are depicted. Contributions estimated using quantified background sources (not upper limits) are marked with asterisk

the rest of this section; the rates are summarized in Table 2 and Fig. 6 and the energy spectra in the low energy region obtained for the dominant quantified internal activities are shown in Fig. 7.

\subsection{Intrinsic radioactivity}

The simulation of the common radioactive isotopes, ${ }^{40} \mathrm{~K}$ and those in the ${ }^{238} \mathrm{U}$ and ${ }^{232} \mathrm{Th}$ chains, from all the main internal components (inside or close to the vessel) has been scaled by the measured activities. Table 3 presents the individual contribution of each of the isotopes in the case of the considered Ne mixture (analogous results have been obtained for the Ar mixture), to identify the most relevant contributor for each simulated component of the set-up. As shown in Table 2, most of the screened components finally used in the set-up have been considered; it is worth noting that many of the reported values are indeed upper limits to the estimated background rate as only upper limits have been derived for the activity of the corresponding element. The thorough selection process of components and materials (described in Sect. 4) has allowed to reduce to non-relevant levels the contributions of, for instance, PTFE components, field cage resistors or silicone connectors.
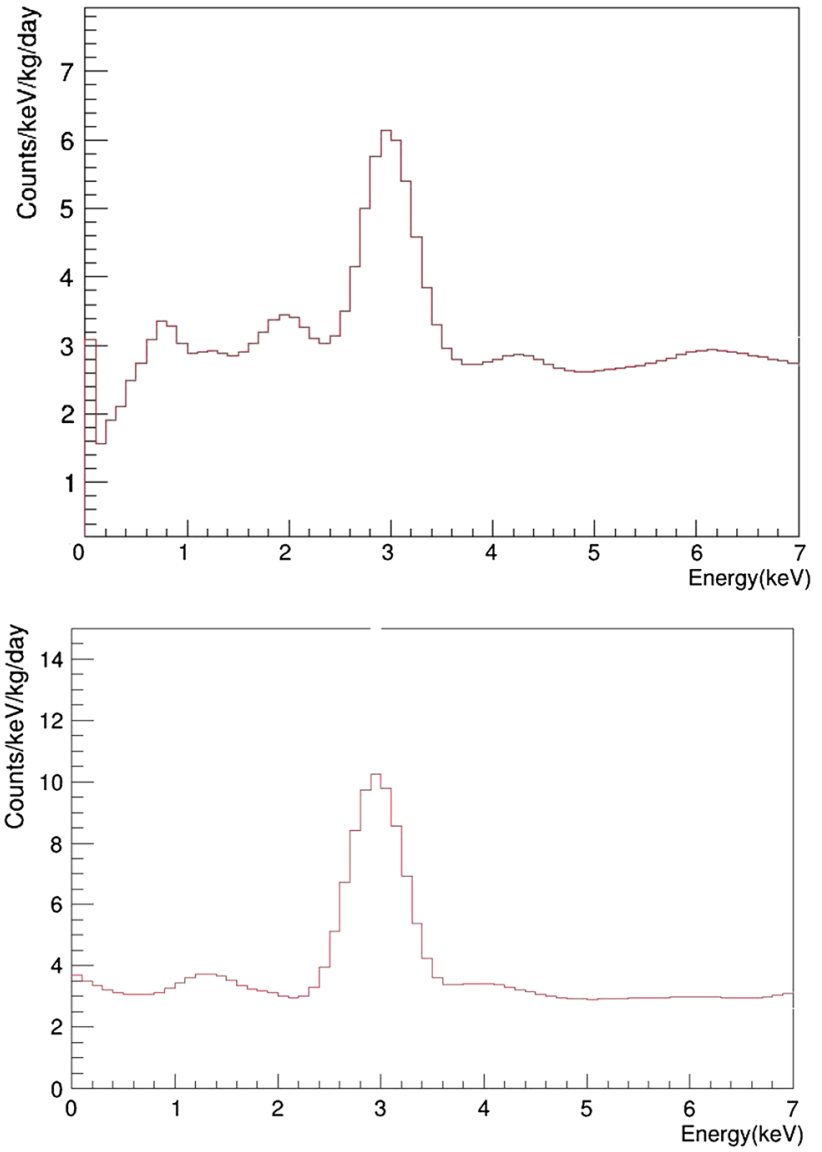

Fig. 7 Background spectra in the low energy region for Ar (top) and $\mathrm{Ne}$ (bottom) mixtures in TREX-DM, simulated for the dominant quantified internal activities: ${ }^{40} \mathrm{~K}$ from the readout planes and cosmogenic ${ }^{60} \mathrm{Co}$ from the copper vessel. The experimental energy resolution of the detector has been considered to depict the spectra

For the Micromegas readout planes, the measured value for ${ }^{40} \mathrm{~K}$ activity before any special cleaning (as in \#31 of Table 1) has been taken into account. It gives a significant rate and therefore the studies underway to reduce this activity, probably related to the process of creating holes, are very important.

\subsection{Cosmogenic activity}

Cosmogenic activation of the set-up materials must be taken into account too. Especially, that of the copper vessel, due to its large mass, and the activation of the gas medium itself.

\subsubsection{Copper}

Production rates of cosmogenic isotopes in copper have been measured [50,51] and evaluated using different codes [5255]; although there is a non-negligible dispersion in results, yields of tens of nuclei per $\mathrm{kg}$ and day are expected for cobalt isotopes having long half-lives (like 271.8 days for ${ }^{57} \mathrm{Co}$, 
Table 3 Background rates (in counts $\mathrm{keV}^{-1} \mathrm{~kg}^{-1}$ day ${ }^{-1}$ ) expected in the RoI $\left(0.2-7 \mathrm{keV}_{e e}\right)$ from the activity of each one of the considered contaminants in components using the Ne mixture in TREX-DM. The numbers with \# at the second column refer to Table 1, indicating the activity values considered. Analogous results have been obtained for the Ar mixture

\begin{tabular}{lllll}
\hline Component & Reference & ${ }^{238} \mathrm{U}$ & ${ }^{232} \mathrm{Th}$ & ${ }^{40} \mathrm{~K}$ \\
\hline Vessel & $\# 2,4$ & $<0.054$ & $<0.034$ & 0.026 \\
Copper Boxes & $\# 2$, see Sect. 5.2.1 & $<0.014$ & $<0.0064$ & $3.7 \times 10^{-4}$ \\
Field Cage (PTFE) & $\# 13$ & $<0.026$ & $<0.026$ & $<7.3 \times 10^{-5}$ \\
Field Cage (resistors) & $\# 9$ & 0.20 & $<0.22$ & 0.053 \\
Field Cage (kapton-Cu PCB) & $\# 7$ & $<0.82$ & $<0.64$ & $<0.087$ \\
Field Cage (cable) & $\# 20$ & $<0.015$ & $<0.018$ & 0.0050 \\
Cathode (copper) & $\# 2$ & $<2.1 \times 10^{-6}$ & $<1.1 \times 10^{-6}$ & $6.8 \times 10^{-7}$ \\
Cathode (PTFE) & $\# 13$ & $<6.7 \times 10^{-5}$ & $<7.1 \times 10^{-5}$ & $<2.3 \times 10^{-7}$ \\
Flat Cables & $\# 19$ & 0.0049 & $<0.0015$ & 0.0021 \\
Connectors & $\# 18$ & 0.0071 & 0.0026 & 0.013 \\
Epoxy & $\# 8$ & $<1.3 \times 10^{-4}$ & $<1.9 \times 10^{-4}$ & $<6.2 \times 10^{-4}$ \\
Mesh Cable & $\# 21$ & $<2.2 \times 10^{-5}$ & $<1.7 \times 10^{-5}$ & $1.4 \times 10^{-4}$ \\
Other PTFE Components & $\# 13$ & $<0.013$ & $<0.013$ & $<3.7 \times 10^{-5}$ \\
Readout Planes & $\# 30,37$ & $<0.36$ & $<0.064$ & 2.3
\end{tabular}

70.85 days for ${ }^{58} \mathrm{Co}$ and 5.271 years for ${ }^{60} \mathrm{Co}$ ). A simulation of the long-lived ${ }^{60} \mathrm{Co}$ emissions from the vessel and copper shields has been carried out. As discussed in Sect. 4.2.1, the cosmogenic activation of the TREX-DM vessel (having a mass of $\sim 0.6$ tons) has been quantified thanks to the screening of a copper sample having the same exposure history; this measured ${ }^{60} \mathrm{Co}$ activity has been considered to evaluate its contribution in the model, which is very relevant (see Table 3). The construction of a new vessel will allow to significantly suppress this contribution. For the inner copper shielding (with a mass of $\sim 2$ tons) and the copper boxes shielding the connectors $(42 \mathrm{~kg})$, the activity corresponding to 3 months of exposure to cosmic rays at sea level and the saturation activity of $(1.000 \pm 0.090) \mathrm{mBq} / \mathrm{kg}$ deduced in [50] has been considered; this contribution is not relevant, as shown in Table 3, thanks not only to the reduced cosmogenic activity but also to the shielding effect provided by the copper vessel for these emissions.

\subsubsection{Gas}

For the specific case of argon-based mixtures, the effect of ${ }^{39} \mathrm{Ar}$, which decays by beta-emission $(\mathrm{Q}=565 \mathrm{keV})$ and has a long half-life (239 years), has been evaluated. It is produced at surface level by cosmogenic activation and the best way to avoid it is extracting argon for underground sources. The lowest activities have been obtained by the DarkSide collaboration using this technique [56]; assuming the measured activity from the DarkSide- 50 data of $0.73 \mathrm{mBq} / \mathrm{kg}$, the contribution to TREX-DM background has been quantified (see Table 2). This value of ${ }^{39} \mathrm{Ar}$ activity means a reduction factor of about 1400 in comparison to the activity in atmospheric argon, which is at the level of $1 \mathrm{~Bq} / \mathrm{kg}$. Therefore, following the estimated contribution in Table 2 for ${ }^{39} \mathrm{Ar}$, it is straightforward to conclude that for operation with atmospheric argon the background level of TREX-DM would be totally dominated by this isotope, giving a rate of 219 counts $\mathrm{keV}^{-1} \mathrm{~kg}^{-1}$ day $^{-1}$ in the RoI. The contribution of ${ }^{85} \mathrm{Kr}$, decaying also by beta-emission $(\mathrm{Q}=687 \mathrm{keV})$ with a halflife of 10.76 years, has been estimated too, considering the measured activity by DarkSide [56], even if cryogenic distillation is expected to help to remove effectively ${ }^{85} \mathrm{Kr}$ from argon.

Among the different radioactive isotopes that can be induced, tritium in the detector medium could be a very relevant background source for a dark matter experiment due to its decay properties: it is a pure beta emitter with $\mathrm{Q}=18.591 \mathrm{keV}$ and a long half-life of 12.312 years. Following the shape of the beta spectrum for the super-allowed transition of ${ }^{3} \mathrm{H}, 57 \%$ of the emitted electrons are in the range from 1 to $7 \mathrm{keV}$; these electrons are fully absorbed in most of the, typically large, dark matter detectors. Some recent studies on tritium production in materials of interest for dark matter experiments can be found in $[52,55,57]$; there are some estimates in argon but no information for neon, and therefore a calculation of production rates in these two targets (assuming their natural isotopic abundances) has been attempted, as presented in [58].

The available information on the excitation function by nucleons has been firstly collected, as shown in Fig. 8: only one experimental result was found in the EXFOR database [59] from [60] and cross sections were taken from the 

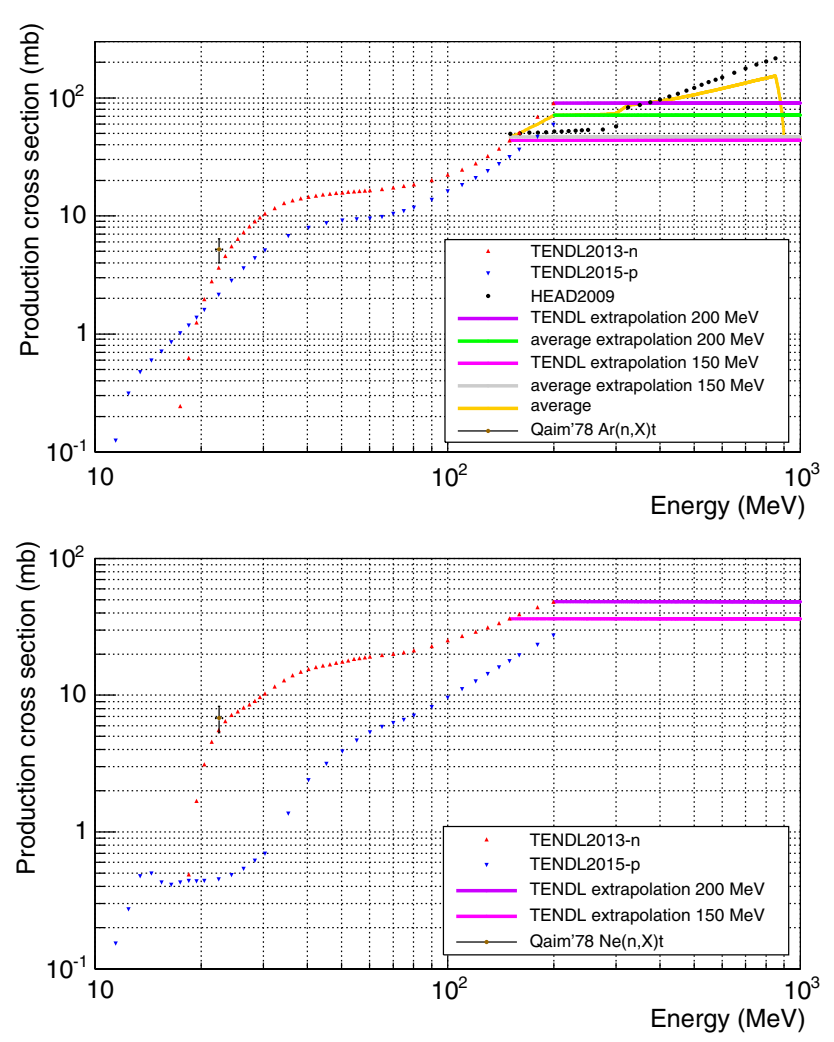

Fig. 8 Comparison of excitation functions for the production of ${ }^{3} \mathrm{H}$ by nucleons for $\mathrm{Ar}$ (top) and $\mathrm{Ne}$ (bottom) taken from different sources: TENDL-2013 and HEAD-2009 libraries together with several extrapolations considered at high energies; the extrapolation of the average between the highest energy cross-section from TENDL and the corresponding results from HEAD-2009 is labeled as "average extrapolation" and the average at each energy between TENDL data (and its extrapolation) and HEAD-2009 cross sections as "average"

TENDL-2013/2015 (TALYS-based Evaluated Nuclear Data Library) library [61] up to $150 / 200 \mathrm{MeV}$ and from the HEAD2009 (High Energy Activation Data) library [62] for higher energies up to $1000 \mathrm{MeV}$. Above $1 \mathrm{GeV}$, a constant production cross-section from the last available energy has been assumed. For Ar, the excitation functions from the two different libraries used at low and high energies match reasonably well. Since the HEAD-2009 library does not provide results for $\mathrm{Ne}$, the last available cross-section value from TENDL2013 has been assumed for all the higher energies. Then, the production rate was computed convoluting a selected excitation function with the energy spectrum of cosmic neutrons at sea level, using the parametrization from [63], following the different approaches plotted in Fig. 8 [58]. The maximum and minimum rates obtained in these approaches define an interval, whose central value and half width have been considered as the final results and their uncertainties for the evaluation of the production rates of tritium in each target. Table 4 summarizes the production rates obtained for $\mathrm{Ar}$ and $\mathrm{Ne}$ and presents other results from the literature: the estimates in [52] using TALYS 1.0 code and those in [55] based on GEANT4 simu-
Table 4 Production rates (in $\mathrm{kg}^{-1} \mathrm{day}^{-1}$ ) of ${ }^{3} \mathrm{H}$ at sea level calculated for Ar and Ne from TENDL-2013 and HEAD-2009 libraries as in [58]; other available values taken from the literature are shown for comparison

\begin{tabular}{lllll}
\hline Target & $\begin{array}{l}\text { TENDL-2013+HEAD-2009 } \\
{[58]}\end{array}$ & $\begin{array}{l}\text { TALYS } \\
{[52]}\end{array}$ & $\begin{array}{l}\text { GEANT4 } \\
{[55]}\end{array}$ & $\begin{array}{l}\text { ACTIVIA } \\
{[55]}\end{array}$ \\
\hline $\mathrm{Ar}$ & $146 \pm 31$ & 44.4 & 84.9 & 82.9 \\
$\mathrm{Ne}$ & $228 \pm 16$ & & & \\
\hline
\end{tabular}

lation and ACTIVIA [54]. The rate in Ar only from TENDL2013 library below $150 \mathrm{MeV}$ is $47.7 \mathrm{~kg}^{-1}$ day $^{-1}$, which is in very good agreement with the value obtained in [52], since the library is also based on TALYS code. It is worth noting that the applied procedure to estimate tritium production rates in $\mathrm{Ar}$ and $\mathrm{Ne}$ gives a very good agreement with the measured rates by EDELWEISS and CDMSlite experiments when applied to natural Ge [58].

Tritium emissions are fully absorbed in the gas producing a signal indistinguishable from that of a dark matter interaction. If saturation activity was reached for tritium, according to production rates in Table 4, it would dominate the expected background model with a contribution of 15 and 22 counts $\mathrm{keV}^{-1} \mathrm{~kg}^{-1} \mathrm{day}^{-1}$ for Ar and $\mathrm{Ne}$, respectively, in the RoI. However, tritium is expected to be suppressed by purification of gas and minimizing exposure to cosmic rays of the purified gas should avoid any problematic tritium activation. The first experimental data in TREX-DM will be extremely useful to confirm that tritium production is not a relevant background source for the experiment.

On the other hand, in TREX-DM, mixtures of $\mathrm{Ar}$ or $\mathrm{Ne}$ with $1-2 \% \mathrm{iC}_{4} \mathrm{H}_{10}$ at $10 \mathrm{~b}$ are foreseen; tritium could be not only cosmogenically induced in the noble gas but also be present in the isobutane. No specific information about tritium content in isobutane has been found. Assuming concentration as in water ${ }^{4}$, this would also give a very relevant contribution in the RoI of TREX-DM of 22 and 84 counts $\mathrm{keV}^{-1} \mathrm{~kg}^{-1} \mathrm{day}^{-1}$ for the considered Ar and Ne mixtures, respectively. In any case, the obtention of isobutane from underground gas sources, shielded from cosmic rays, avoids a dangerous tritium content.

\subsection{Radon-induced activity}

A simulation of the ${ }^{222} \mathrm{Rn}$ in the air surrounding the vessel inside the copper shielding has been performed. The measured activity at hall A of LSC of $(63 \pm 1) \mathrm{Bq} / \mathrm{m}^{3}[44]$ has been considered; the implementation of a $\mathrm{N}_{2}$ gas flux system

\footnotetext{
${ }^{4}$ For natural surface waters there are about one tritium atom per $10^{18}$ atoms of hydrogen, following Ref. [64]. The measured tritium activity is some waters and the limits for drinking water give indeed higher concentrations.
} 
inside the shielding to avoid radon intrusion providing a factor $\sim 100$ reduction in the air ${ }^{222} \mathrm{Rn}$ activity allows to reduce this contribution to a non-relevant level (see Table 2). Even if radon inside the shielding has not been directly quantified, the reduction factor assumed here and in other experiments operated in Canfranc with similar shielding and nitrogen flux is supported by the good agreement between models and measured data for peaks due to ${ }^{222} \mathrm{Rn}$ emissions [24]. In any case, this assumption is not very relevant because if TREXDM operates using the radon-free air facility available at the LSC a bigger radon suppression factor at the level of about 1000 would be at reach.

An estimate of the contribution to the TREX-DM background of a long exposure to air-borne radon of components of the set-up has been attempted, evaluating the effect of the creation of a long-lived ${ }^{210} \mathrm{~Pb}$ contamination and its progeny. This study has been carried out for the copper vessel, having a large surface $\left(\sim 1.4 \mathrm{~m}^{2}\right)$ exposed to a normal atmosphere for a long time, and for the inner part of the copper shielding $\left(\sim 2.9 \mathrm{~m}^{2}\right)$. For the induced ${ }^{210} \mathrm{~Pb}$ activity on the copper surface, the upper limit set from the screening of a sample of the vessel material described in Sect. 4.2.1 has been considered. It is worth noting that the considered limit on ${ }^{210} \mathrm{~Pb}$ is $\sim 19$ times larger than the measured ${ }^{210} \mathrm{Po}$ surface activity on an ETP copper exposed to radon for years [65] and $\sim 10-30$ times larger than the calculated activity assuming 2 years of exposure to an activity of $15 \mathrm{~Bq} / \mathrm{m}^{3}$ of ${ }^{222} \mathrm{Rn}$ following the results in [66,67]; therefore, it is considered a very conservative value. Emissions of ${ }^{210} \mathrm{~Pb}$ from the copper surfaces have been simulated and, following results in Table 2, the contribution is not worrisome.

\subsection{Gamma background}

The environmental gamma flux contribution from ${ }^{238} \mathrm{U}$, ${ }^{232} \mathrm{Th}$ and ${ }^{40} \mathrm{~K}$ emissions has been evaluated by simulating the corresponding photons through the whole TREX-DM set-up and scaling by the measured flux in hall A of LSC, as reported in [68]. Due to lack of statistics, modest upper limits for the counting rate in the $\mathrm{RoI}\left(0.2-7 \mathrm{keV}_{e e}\right)$ at the level of 10-20 counts $\mathrm{keV}^{-1} \mathrm{~kg}^{-1}$ day $^{-1}$ ) have been set from this simulation. But the $20-\mathrm{cm}$-thick lead shielding together with the additional $11 \mathrm{~cm}$ copper layer from vessel and inner shielding allows for a reduction of several orders of magnitude of the environmental gamma flux and this contribution can be safely neglected.

\subsection{Muons}

The muon contribution has been simulated assuming the measured muon flux at LSC of $\sim 4 \times 10^{-3} \mathrm{~s}^{-1} \mathrm{~m}^{-2}$, as in [69]. The muon energy spectrum evaluated for the Canfranc depth (with mean energy of $216 \mathrm{GeV}$ ) and the corre- sponding angular distribution have been considered following [70]. Muons are launched from a virtual wall through the whole TREX-DM set-up. The counting rate derived in the RoI without applying any discrimination method is high, but following the previous results derived for TREX-DM by analyzing the signal topology in [14], only 5.4 (3.4)\% of events survive the cuts for Argon (Neon); this makes the muon contribution non-dominant (see Table 2), even without the implementation of a muon veto. It must be noted that the production of neutrons by muons is implemented in the simulation and therefore the contribution of these high energy neutrons produced in the lead shielding is included in the background level reported for muons.

\subsection{Neutrons}

The effect of neutrons from other origins has been analyzed for TREX-DM too. First, environmental neutrons at LSC have been simulated from a virtual sphere containing the set-up with the 40-cm-thick moderator and the results have been normalized to the neutron flux measured at LSC in [71] (similar to that presented in [72]); the evaporation spectrum has been considered for the neutron energy sampling. A preliminary analysis of the tracks left in the chamber points to a rejection factor of $\sim 10 \%$ of the events in the RoI. The obtained rates are shown in Table 2; the neutron moderator makes the contribution from these neutrons irrelevant. Concerning muon-induced neutrons in the rock surrounding the laboratory, the expected flux is $\sim 3$ orders of magnitude lower than that of the environmental neutrons [72].

Radiogenic neutrons produced by $(\alpha, \mathrm{n})$ reactions due to ${ }^{238} \mathrm{U}$ and ${ }^{232} \mathrm{Th}$ primordial impurities (or spontaneous fission of the former) in the set-up materials are other of the neutron sources of background in WIMP direct detection experiments. In particular, neutrons having also an evaporation spectrum have been simulated from the lead shielding and the copper vessel to evaluate the contribution from the fission of their ${ }^{238} \mathrm{U}$ contaminations. The information on this isotope activity for copper and lead presented in Table 1 has been considered together with the values of $(2.4 \pm 0.2)$ neutrons per fission and $(5.45 \pm 0.04) \times 10^{-7}$ fissions per decay [73]. The deduced counting rates, shown in Table 2, are negligible.

Additionally, an estimate of the contribution of these radiogenic neutrons in other materials of the TREX-DM setup has been attempted using a simulation of neutrons having a fission energy spectrum and calculating the expected neutron rate from neutron yields estimated in previous works for the materials. In particular, neutron yields for copper, steel, teflon and polyethylene from [74] have been used; yields for $\mathrm{Ar}$ and $\mathrm{Ne}$ are available in [75]. Results in [74] were obtained using the SOURCES4A code with $(\alpha, \mathrm{n})$ reaction cross-sections calculated using EMPIRE2.19, while calcula- 
Table 5 Estimates of the expected counting rate in TREX-DM using Ar or Ne from radiogenic neutrons produced by uranium and thorium presence in the main materials of the set-up

\begin{tabular}{|c|c|c|c|c|c|c|}
\hline & $\begin{array}{l}\text { Neutron yield } \\
\left(\mathrm{ppm}^{-1} \mathrm{~g}^{-1} \text { year }^{-1}\right)\end{array}$ & Mass (kg) & Concentration (ppm) & $\begin{array}{l}\text { Total yield (neu- } \\
\text { trons day }{ }^{-1} \text { ) }\end{array}$ & $\begin{array}{l}\text { Rate in } \mathrm{Ar} \text { (counts } \\
\left.\mathrm{keV}^{-1} \mathrm{~kg}^{-1} \mathrm{day}^{-1}\right)\end{array}$ & $\begin{array}{l}\text { Rate in } \mathrm{Ne} \text { (counts } \\
\mathrm{keV}^{-1} \mathrm{~kg}^{-1} \text { day }^{-1} \text { ) }\end{array}$ \\
\hline U, copper & 0.436 & 997.3 & $<1.010^{-6}$ & $<1.210^{-3}$ & $<8.910^{-7}$ & $<1.710^{-6}$ \\
\hline Th, copper & 0.030 & 997.3 & $<1.010^{-6}$ & $<8.210^{-5}$ & $<6.210^{-8}$ & $<1.210^{-7}$ \\
\hline U, PTFE & 26.2 & 3.96 & $<5.010^{-6}$ & $<1.410^{-3}$ & $<1.110^{-6}$ & $<2.110^{-6}$ \\
\hline Th, PTFE & 11.23 & 3.96 & $<1010^{-6}$ & $<1.210^{-3}$ & $<9.110^{-7}$ & $<1.810^{-6}$ \\
\hline $\mathrm{U}$, steel & 0.587 & 920 & $2.610^{-3}$ & 3.8 & $4.810^{-4}$ & $9.110^{-4}$ \\
\hline Th, steel & 0.189 & 920 & $1.110^{-3}$ & $5.510^{-1}$ & $6.910^{-5}$ & $1.310^{-4}$ \\
\hline $\mathrm{U}$, polyethylene & 0.894 & 3055 & $<5.010^{-6}$ & $<3.710^{-2}$ & $<4.710^{-6}$ & $<8.910^{-6}$ \\
\hline Th, polyethylene & 0.193 & 3055 & $<5.010^{-6}$ & $<8.110^{-3}$ & $<1.010^{-6}$ & $<1.910^{-6}$ \\
\hline Total & & & & & $<5.610^{-4}$ & $<1.110^{-3}$ \\
\hline
\end{tabular}

tions in [75] are based on cross-sections obtained with the TALYS code. Secular equilibrium in the natural chains is assumed in both references. Yields in [74] include also spontaneous fission of ${ }^{238} \mathrm{U}$. In [75] it is reported that there is no $(\alpha, \mathrm{n})$ neutron yield in lead due to a very high Coulomb barrier. A code (NeuCBOT) for computation of neutron yields and the corresponding estimates for different materials have been presented too in [76]; it is based on TALYS for cross sections and uses ENSDF for alpha decay data and SRIM code for considering the stopping power of alpha particles. It is reported that NeuCBOT tends to predict yields systematically higher by $\sim 30 \%$ than other calculations based on SOURCES4A or available measured yields. Table 5 summarizes the considered neutron yields and the corresponding estimated counting rates in TREX-DM. The whole mass of each material in the set-up has been considered: copper in vessel, small components and shielding structure, teflon from field cage and other components, steel in the shielding structure and polyethylene used as neutron moderator. The following activities for ${ }^{238} \mathrm{U}$ and ${ }^{232} \mathrm{Th}$ (using the highest value of those corresponding to the different isotopes in the chain, if available) have been assumed: the deduced values for TREXDM for copper and teflon (\#2 and \#13 in Table 1), measured values for $\mathrm{S} 275$ steel (as used in TREX-DM shielding structure) by NEXT [47] and upper limits derived by ICPMS for both $\mathrm{U}$ and $\mathrm{Th}$ in polyethylene (from a different supplier) by NEXT [77]. As shown in Table 5, neutrons from steel are the most relevant; the upper limit to the total estimated contribution to the background rate is of the order of $10^{-3}$ counts $\mathrm{keV}^{-1} \mathrm{~kg}^{-1}$ day $^{-1}$, and therefore, it can be concluded that radiogenic neutrons are not a dominant background source for TREX-DM. This contribution is also shown in Table 2. No reference of $U$ and Th content in the gases (Ar or Ne) is available; but it has been checked, using the yields in [75], that concentrations of $\mathrm{U}$ and $\mathrm{Th}$ about $1 \mathrm{ppm}$ would be required to produce rates at the level of $10^{-3}$ counts $\mathrm{keV}^{-1} \mathrm{~kg}^{-1}$ day ${ }^{-1}$ comparable to those from the other materials.
The contribution from muons and environmental neutrons is under control in the simulated conditions, following the rates in Table 2. Although the precise estimates of some external background sources are still underway, it seems that the designed shielding is enough to reduce to non-relevant levels the corresponding contribution.

\section{Sensitivity prospects}

For completeness, a first estimate of the achievable sensitivity in the search for low mass WIMPs is presented in this section in order to assess the effect of the background conditions evaluated and the expected detector performance. Figure 9 presents the attainable exclusion plots (90\% CL) in the direct detection of WIMPs, for both $\mathrm{Ar}$ and Ne-based gas mixtures at 10 bar, obtained assuming spin independent (SI) interaction and standard values of the WIMP halo model with Maxwell-Boltzmann velocity distribution and astrophysical parameters (local dark matter density $0.3 \mathrm{GeV} / \mathrm{c}^{2}$, local velocity $220 \mathrm{~km} / \mathrm{s}$, laboratory velocity $232 \mathrm{~km} / \mathrm{s}$ and galactic escape velocity $544 \mathrm{~km} / \mathrm{s}$ ). The projected exclusion curves have been derived as in [14], assuming the background is properly accounted for by the background model and statistically subtracted. Three different scenarios (labeled as A, B and C) for flat-shaped background, energy threshold and exposure have been considered (see Table 6). Despite the peak at $\sim 3 \mathrm{keV}$ appearing in the RoI due to the ${ }^{40} \mathrm{~K}$ activity in the readout planes (see Fig. 7), the flat background assumption is reasonable as the exclusion plot is mainly determined by the lowest energy bins just above the energy threshold; in addition, works are underway to suppress this background contribution. A data-taking campaign of approximately 3 years is foreseen, starting with $\mathrm{Ne}$ with the option to change to depleted Ar. It can be seen that even in the first stage of TREX-DM with a small-scale set-up there is an option to explore regions of WIMPs uncovered by other experiments. 


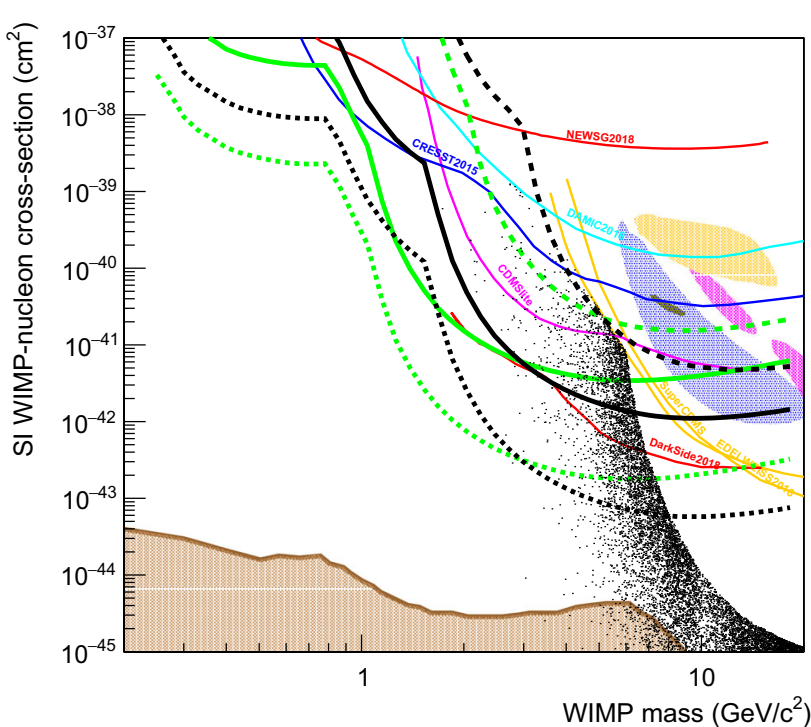

Fig. 9 90\% CL sensitivity of TREX-DM under three different conditions for background level, energy threshold and exposure, summarized in Table 6 , for $\mathrm{Ar}+1 \% \mathrm{iC}_{4} \mathrm{H}_{10}$ (black lines) and $\mathrm{Ne}+2 \% \mathrm{iC}_{4} \mathrm{H}_{10}$ (green lines). The closed contours shown correspond to results from CDMS-II Si [78] (blue, 90\% CL), CoGeNT [2] (dark gray, 90\% CL), CRESST-II [79] (magenta, 95\% CL), and DAMA/LIBRA [80] (yellow, 90\% CL). The brown shaded region corresponds to the sensitivity limit imposed by the solar neutrino coherent scattering background [81]. As a reference, 90\% CL exclusion limits from different experiments are also shown: CRESST-II 2016 [7] (dark blue), CDMSlite [5,6] (purple), DAMIC [3] (light blue), EDELWEISS [9] and SuperCDMS [82] (yellow) and DarkSide [12] and NEWS-G [13] (red)

Table 6 Conditions assumed in the calculations of the TREX-DM sensitivity shown in figure 9

\begin{tabular}{|c|c|c|c|}
\hline & A & $\mathrm{B}$ & $\mathrm{C}$ \\
\hline Background level (counts $\mathrm{keV}^{-1} \mathrm{~kg}^{-1} \mathrm{day}^{-1}$ ) & 10 & 1 & 0.1 \\
\hline Energy threshold $\left(\mathrm{keV}_{e e}\right)$ & 0.4 & 0.1 & 0.1 \\
\hline Exposure $(\mathrm{kg}$ y) & 0.3 & 0.3 & 10 \\
\hline
\end{tabular}

Following the rates summarized in Table 2, the background assumed in scenario A gives the worst expectation. To achieve the level considered in scenario $\mathrm{B}$, a reduction of the two main contributions from the quantified present activities should be enough. On one hand, as discussed in Sect. 4.2.4, work is underway to reduce the ${ }^{40} \mathrm{~K}$ content of the microbulk Micromegas readout and from the available results a reduction factor of $\sim 4$ has been already achieved (see Table 1). On the other hand, a new copper vessel produced using fresh copper and limiting exposure to cosmic rays at sea level to 1 month would have a ${ }^{60} \mathrm{Co}$ activity of $0.01 \mathrm{mBq} / \mathrm{kg}$ (from the saturation activity deduced in [50]), giving a rate of $0.06(0.07)$ counts $\mathrm{keV}^{-1} \mathrm{~kg}^{-1} \mathrm{day}^{-1}$ for $\operatorname{Ar}(\mathrm{Ne})$, which would mean a reduction by a factor 22 respect to the present copper vessel. The scenario B is interesting for
$\mathrm{Ne}$ as for WIMP masses below $2 \mathrm{GeV} / \mathrm{c}^{2}$ the sensitivity is beyond current bounds.

The background level considered in scenario $\mathrm{C}$, predicting the stringent exclusion plots, appears as a plausible future goal. Several individual contributions are at the level of 0.1 counts $\mathrm{keV}^{-1} \mathrm{~kg}^{-1} \mathrm{day}^{-1}$ : those of connectors, radon in air, muons and ${ }^{39} \mathrm{Ar}$ for Ar. New connectors already screened (see results at \#19 of Table 1) would guarantee more than one order of magnitude of reduction for this contribution. Moreover, this background contribution could be totally suppressed if the new connection approach being tested is successful; multi-channel connectors can be replaced by a new custom-made "face-to-face" contact technique offering in addition a substantial improvement in the level of connectivity. This system has been designed, manufactured, installed and it is presently being checked. The use of a muon veto system or a specific, more efficient, muon discrimination analysis together with the use of air from the radon-free air factory in operation in Canfranc offering an overall reduction factor of $\sim 1000$ in the radon activity would also help to reduce these external contributions.

\section{Conclusions}

The TREX-DM experiment intends to look for low mass WIMPs using a Micromegas-read High Pressure TPC filled with Ar or Ne mixtures in the Canfranc Underground Laboratory. At the beginning of 2019 , it is at the commissioning phase and the data taking is expected to start soon using $\mathrm{Ne}+2 \% \mathrm{iC}_{4} \mathrm{H}_{10}$. Together with a sub-keV $\mathrm{K}_{e e}$ energy threshold, an ultra-low background level at the lowest energy region is mandatory; an assessment of the expected background has been performed to help in the selection of radiopure components during the design phase and to support a reliable estimate of the experiment sensitivity. The background contributions, taking into consideration all the known sources, have been simulated by means of a dedicated Geant 4 application and a custom-made code implementing the detector response.

A material radioassay campaign has been carried out, based on germanium spectrometry at LSC and complementary measurements, to quantify the activity of all the relevant elements in the experiment set-up (see Table 1). The total expected background level from the internal activity should be below 6.1(6.6) counts $\mathrm{keV}^{-1} \mathrm{~kg}^{-1}$ day $^{-1}$ for $\operatorname{Ar}(\mathrm{Ne})$, as shown in Table 2; from that, 70(64)\% come from activities actually quantified. One of the largest contributions is due to the copper vessel, cosmogenically activated after being a few years at sea level, as shown in a dedicated germanium measurement. This important contribution could be suppressed by constructing a new vessel. The measured ${ }^{40} \mathrm{~K}$ activity in the Micromegas readout gives also a significant 
rate and for this reason new treatments are being analyzed in an attempt to reduce this activity. It must be noted that the use of underground argon has been assumed, considering the ${ }^{39} \mathrm{Ar}$ activity measured by DarkSide [56]. It has been verified that a saturation activity of tritium in the gas media could be very relevant, but the gas purification and obtention from underground sources will avoid in principle this contribution. The effect of radon and radon-induced activity on copper surfaces has been assessed, finding it non-dominant at the present phase. The contribution from muons and environmental neutrons is under control in the simulated conditions, thanks to the background rejection capabilities and the shielding. All in all, in the presently assumed conditions, the TREX-DM expected background can be considered between 1 and 10 counts $\mathrm{keV}^{-1} \mathrm{~kg}^{-1} \mathrm{day}^{-1}$; a few improvements have been identified and are being tested in an attempt to further decrease it down to 0.1 counts $\mathrm{keV}^{-1} \mathrm{~kg}^{-1}$ day ${ }^{-1}$, which would provide a competitive sensitivity in the direct detection of low mass WIMPs.

Acknowledgements This work has been financially supported by the European Commission under the European Research Council T-REX Starting Grant ref. ERC-2009-StG-240054 of the IDEAS program of the 7th EU Framework Program and by the Spanish Ministry of Economy and Competitiveness (MINECO) under Grants FPA2013-41085-P and FPA2016-76978-C3-1-P. We thank Rui de Oliveira from the EP-DT-DD Micro-Pattern Technologies (MPT) service at CERN for the preparation and cleaning of the Micromegas samples and the CEA/Saclay collaborators for their assistance. We also acknowledge LSC and GIFNA staff for their support.

Data Availability Statement This manuscript has no associated data or the data will not be deposited. [Authors' comment: The TREX-DM experiment has not started the data taking for dark matter searches yet.]

Open Access This article is distributed under the terms of the Creative Commons Attribution 4.0 International License (http://creativecomm ons.org/licenses/by/4.0/), which permits unrestricted use, distribution, and reproduction in any medium, provided you give appropriate credit to the original author(s) and the source, provide a link to the Creative Commons license, and indicate if changes were made. Funded by SCOAP $^{3}$.

\section{References}

1. T. Marrodan-Undagoitia, L. Rauch, Dark matter direct-detection experiments. J. Phys. G 43, 013001 (2016)

2. C.E. Aalseth et al., CoGeNT: a search for low-mass dark matter using p-type point contact germanium detectors. Phys. Rev. D 88, 012002 (2013)

3. A. Aguilar-Arevalo et al., Search for low-mass WIMPs in a $0.6 \mathrm{~kg}$ day exposure of the DAMIC experiment at SNOLAB. Phys. Rev. D 94, 082006 (2016)

4. L.T. Yang et al., Limits on light WIMPs with a $1 \mathrm{~kg}$-scale germanium detector at 160 eVee physics threshold at the China Jinping Underground Laboratory. Chin. Phys. C 42, 23002 (2018)
5. R. Agnese et al., New Results from the Search for Low-Mass Weakly Interacting Massive Particles with the CDMS Low Ionization Threshold Experiment. Phys. Rev. Lett. 116, 071301 (2016)

6. R. Agnese et al., Low-mass dark matter search with CDMSlite. Phys. Rev. D 97, 022002 (2018)

7. G. Angloher et al., Results on light dark matter particles with a low-threshold CRESST-II detector. Eur. Phys. J. C 76, 25 (2016)

8. A.H. Abdelhameed et al., First results from the CRESST-III lowmass dark matter program, arXiv:1904.00498 [astro-ph.CO]

9. L. Hehn et al., Improved EDELWEISS-III sensitivity for low-mass WIMPs using a profile likelihood approach. Eur. Phys. J C 76, 548 (2016)

10. Q. Arnaud et al., Optimizing EDELWEISS detectors for low-mass WIMP searches. Phys. Rev. D 97, 022003 (2018)

11. E. Aprile et al. (XENON100 Collaboration), Low-mass dark matter search using ionization signals in XENON100, Phys. Rev. D 94, 092001 (2016). Erratum: Phys. Rev. D 95, 059901 (2017)

12. P. Agnes et al., (The DarkSide Collaboration), Low-mass dark matter search with the DarkSide-50 experiment. Phys. Rev. Lett. 121, 081307 (2018)

13. Q. Arnaud et al., (NEWS-G Collaboration), First results from the NEWS-G direct dark matter search experiment at the LSM. Astropart. Phys. 97, 54 (2018)

14. F.J. Iguaz et al., TREX-DM: a low-background Micromegas-based TPC for low-mass WIMP detection. Eur. Phys. J. C 76, 529 (2016)

15. I.G. Irastorza et al., Gaseous time projection chambers for rare event detection: results from the T-REX project. II. Dark matter. JCAP 01, 034 (2016)

16. S. Andriamonje et al., Development and performance of Microbulk Micromegas detectors. JINST 5, P02001 (2010)

17. I.G. Irastorza et al., Gaseous time projection chambers for rare event detection: results from the T-REX project. I. Double beta decay. JCAP 01, 033 (2016)

18. E. Aprile et al., Study of the electromagnetic background in the XENON100 experiment. Phys. Rev. D 83, 082001 (2011)

19. H.M. Araujo et al., Radioactivity backgrounds in ZEPLIN-III. Astropart. Phys. 35, 495 (2012)

20. E. Armengaud et al., Background studies for the EDELWEISS dark matter experiment. Astropart. Phys. 47, 1 (2013)

21. M. Agostini et al., The background in the neutrinoless double beta decay experiment GERDA. Eur. Phys. J. C 74, 2764 (2014)

22. D.S. Akerib et al., Radiogenic and Muon-Induced Backgrounds in the LUX Dark Matter Detector. Astropart. Phys. 62, 33 (2015)

23. J.B. Albert et al., Investigation of radioactivity-induced backgrounds in EXO-200. Phys. Rev. C 92, 015503 (2015)

24. J. Amare et al., Assessment of backgrounds of the ANAIS experiment for dark matter direct detection. Eur. Phys. J. C 76, 429 (2016)

25. C. Alduino et al., (CUORE Collaboration), The projected background for the CUORE experiment. European Physical Journal C 77, 543 (2017)

26. G. Adhikari et al., Understanding $\mathrm{NaI}(\mathrm{Tl})$ crystal background for dark matter Searches. Eur. Phys. J. C 77, 437 (2017)

27. P. Adhikari et al., Background model for the $\mathrm{NaI}(\mathrm{Tl})$ crystals in COSINE-100. Eur. Phys. J. C 78, 490 (2018)

28. F. Aznar et al., Status of the TREX-DM experiment at the Canfranc Underground Laboratory, To appear at the Proceedings of TAUP2017 at Journal of Physics (Conference Series), arXiv:1709.10062

29. F.J. Iguaz et al., Microbulk Micromegas in non-flammable mixtures of argon and neon at high pressure, in preparation (2019)

30. S. Cebrian et al., Micromegas-TPC operation at high pressure in xenon-trimethylamine mixtures. JINST 8, P01012 (2013)

31. S. Agostinelli et al., Geant4: a simulation toolkit. Nucl. Instrum. Method A 506, 250 (2003)

32. https://sultan.unizar.es/rest/. Accessed 16 Sept 2019 
33. X. Chen et al., PandaX-III: searching for neutrinoless double beta decay with high pressure ${ }^{136} \mathrm{Xe}$ gas time projection chambers. Sci. Chin. Phys. Mech. Astron. 60, 061011 (2017)

34. G. Cirrone et al., Validation of the Geant4 electromagnetic photon cross-sections for elements and compounds. Nucl. Instrum. Methods A 618, 315 (2010)

35. F. Aznar et al., Assessment of material radiopurity for rare event experiments using micromegas. JINST 8, C11012 (2013)

36. E. Aprile et al., Material radioassay and selection for the XENON1T dark matter experiment. Eur. Phys. J. C 77, 890 (2017)

37. D.S. Leonard et al., Trace radioactive impurities in final construction materials for EXO-200. Nucl. Instrum. Methods A 871, 169 (2017)

38. N. Abgrall et al., The Majorana Demonstrator Radioassay Program. Nucl. Instrum. Methods A 828, 22 (2016)

39. V. Alvarez et al., Radiopurity control in the NEXT-100 double beta decay experiment: procedures and initial measurements. JINST 8, T01002 (2013)

40. S. Cebrian et al., Radiopurity assessment of the tracking readout for the NEXT double beta decay experiment. JINST 10, P05006 (2015)

41. S. Cebrian et al., Radiopurity assessment of the energy readout for the NEXT double beta decay experiment. JINST 12, T08003 (2017)

42. J.C. Loach et al., A database for storing the results of material radiopurity measurements. Nucl. Instrum. Methods A 839, 6(2016)

43. S. Cebrian et al., Radiopurity of Micromegas readout planes. Astropart. Phys. 34, 354 (2011)

44. I. Bandac et al., Ultra-low background and environmental measurements at Laboratorio Subterraneo de Canfranc (LSC). Appl. Radiat. Isot. 126, 127 (2017)

45. S. Nisi et al., ICP-MS measurement of natural radioactivity at LNGS. Int. J. Mod. Phys. A 32, 1743003 (2017)

46. A.S. Barabash et al., The BiPo-3 detector for the measurement of ultra low natural radioactivities of thin materials. JINST 12, P06002 (2017)

47. S. Cebrian et al., Radon and material radiopurity assessment for the NEXT double beta decay experiment. AIP Conf. Proc. 1672, 060002 (2015)

48. S. Cebrian, Cosmogenic activation of materials. Int. J. Mod. Phys. A 32, $1743006(2017)$

49. P.F. Smith, J.D. Lewin, Dark matter detection. Phys. Rept. 187, 203 (1990)

50. M. Laubenstein, G. Heusser, Cosmogenic radionuclides in metals as indicator for sea level exposure history. Appl. Radiat. Isot. 67, 750 (2009)

51. L. Baudis et al., Cosmogenic activation of xenon and copper. Eur. Phys. J. C 75, 485 (2015)

52. D.M. Mei et al., Cosmogenic production as a background in searching for rare physics processes. Astropart. Phys. 31, 417 (2009)

53. S. Cebrian et al., Cosmogenic activation in germanium and copper for rare event searches. Astropart. Phys. 33, 316 (2010)

54. J.J. Back, Y.A. Ramachers, ACTIVIA: calculation of isotope production cross-sections and yields. Nucl. Instrum. Methods A 586, $286(2008)$

55. C. Zhang et al., Cosmogenic activation of materials used in rare event search experiments. Astropart. Phys. 84, 62 (2016)

56. P. Agnes et al., Results from the first use of low radioactivity argon in a dark matter search. Phys. Rev. D 93, 081101(R) (2016)

57. E. Armengaud et al., Measurement of the cosmogenic activation of germanium detectors in EDELWEISS-III. Astropart. Phys. 91, 51 (2017)

58. J. Amare et al., Cosmogenic production of tritium in dark matter detectors. Astropart. Phys. 97, 96 (2018)
59. Experimental Nuclear Reaction Data (EXFOR) http://www.nndc. bnl.gov/exfor/exfor.htm, http://www-nds.iaea.org/exfor/exfor. htm. Accessed 16 Sept 2019

60. S.M. Qaim, R. Wolfle, Triton emission in the interactions of fast neutrons with nuclei. Nucl. Phys. A 295, 150 (1978)

61. A.J. Koning, D. Rochman, Modern Nuclear Data Evaluation With The TALYS Code System, Nuclear Data Sheets 113, 2841 (2012). http://www.talys.eu/tendl-2013.html. Accessed 16 Sept 2019

62. Y.A. Korovin et al., High energy activation data library (HEAD2009). Nucl. Instrum. Methods A 624, 20 (2010)

63. M.S. Gordon et al, Measurement of the Flux and Energy Spectrum of Cosmic-Ray Induced Neutrons on the Ground, IEEE Trans. Nucl. Sci. 51, 3427 (2004). Erratum: M. S. Gordon et al., IEEE Transactions on Nuclear Science 52, 2703 (2005)

64. D.G. Jacobs, Sources of tritium and its behaviour upon release to the environment, AEC Critical Review Series, 1968, https://www. osti.gov/biblio/4799828. Accessed 16 Sept 2019

65. G. Zuzel et al., Studies of surface and bulk ${ }^{210} \mathrm{Po}$ in metals using an ultra-low background large surface alpha spectrometer. Appl. Radiat. Isot. 126, 165 (2017)

66. M. Stein et al., Radon daughter plate-out measurements at SNOLAB for polyethylene and copper. Nucl. Instrum. Methods A 880 , 92 (2018)

67. M. Clemenza et al., Radon-induced surface contaminations in low background experiments. Eur. Phys. J. C 71, 1805 (2011)

68. J. Martin-Albo et al., Sensitivity of NEXT-100 to neutrinoless double beta decay. JHEP 05, 15 (2016)

69. G. Luzon et al., Characterization of the Canfranc Underground Laboratory: status and future plans, Proceedings of the 6th International Workshop on The Identification of Dark Matter (IDM 2006), 514 (2006)

70. P.K.F. Grieder, Cosmic rays at earth (Ed. Elsevier, Amsterdam, 2001)

71. D. Jordan et al., Measurement of the neutron background at the Canfranc Underground Laboratory LSC. Astropart. Phys. 42, 1 (2013)

72. J.M. Carmona et al., Neutron background at the Canfranc underground laboratory and its contribution to the IGEX-DM dark matter experiment. Astropart. Phys. 21, 523 (2004)

73. http://www.nucleide.org/DDEP_WG/Nuclides/U-238_tables. pdf. Accessed 16 Sept 2019

74. V. Tomasello et al., Calculation of neutron background for underground experiments. Nucl. Instrum. Methods A 595, 431 (2008)

75. D.-M. Mei et al., Evaluation of $(\alpha, \mathrm{n})$ induced neutrons as a background for dark matter experiments, Nucl. Instrum. Meth. A 606, 651 (2009), http://neutronyield.usd.edu/. Accessed 16 Sept 2019

76. S. Westerdale, P.D. Meyers, Radiogenic neutron yield calculations for low-background experiments. Nucl. Instrum. Methods A 875, 57 (2017)

77. T. Dafni et al., Results of the material screening program of the NEXT experiment. Nucl. Part. Phys. Proc. 273-275, 2666 (2016)

78. R. Agnese et al., Silicon detector dark matter results from the final exposure of CDMS II. Phys. Rev. Lett. 111, 251301 (2013)

79. G. Angloher et al., Results from $730 \mathrm{~kg}$ days of the CRESST-II Dark Matter search. Eur. Phys. J. C 72, 1971 (2012)

80. R. Bernabei et al., Final model independent result of DAMA/LIBRA phase1. Eur. Phys. J. C 73, 2648 (2013)

81. J. Billard, L. Strigari, E. Figueroa-Feliciano, Implication of neutrino backgrounds on the reach of next generation dark matter direct detection experiments. Phys. Rev. D 89, 023524 (2014)

82. R. Agnese et al., Search for low-mass weakly interacting massive particles with SuperCDMS. Phys. Rev. Lett. 112, 241302 (2014) 